Supporting Information

\title{
Strategies to Mitigate the Bioactivation of Aryl Amines
}

Chenghong Zhang, $\uparrow$ James J. Crawford, $\uparrow^{*}$ Matthew L. Landry, Huifen Chen, Jane R. Kenny, S. Cyrus Khojasteh, Wendy Lee, Shuguang Ma, Wendy B. Young

Genentech Inc., One DNA Way, South San Francisco, CA 94080

1. In vitro GSH Trapping Assay Protocol and Associated Data S2

2. Chemical Characterization and Purity S6

3. In Silico Modeling and Associated Data $\quad$ S16

4. Supporting Information References $\quad$ S25 


\section{In vitro GSH Trapping Assay Protocol}

Pooled human liver microsomes (HLM, 200 donor pool) were purchased from Corning Gentest. Reduced $\beta$-nicotinamide adenine dinucleotide phosphate (NADPH) was purchased from Roche Diagnostics (Indianapolis, IN). Dimethylsulfoxide (DMSO), glutathione (GSH) and 3-methylindole were purchased from Sigma-Aldrich (St. Louis, MO). Stable labeled GSH $\left({ }^{13} \mathrm{C}_{2},{ }^{15} \mathrm{~N}\right.$ on glycine) was purchased from Cambridge Isotope Laboratories, Inc. (Andover, MA). HPLC grade acetonitrile (ACN) was purchased from Honeywell Burdick \& Jackson (Muskegon, MI). Water was purified by Purelab Ultra system, ELGA LabWater (High Wycombe, UK). HPLC grade formic acid was purchased from VWR International (West Chester, PA). Potassium phosphate buffer ( $\mathrm{pH}$ 7.4) was prepared by Media Preparation Facility at Genentech Inc. (South San Francisco, CA). All tested chemicals were obtained from commercial sources or were previously synthesized at Genentech.

Incubation: GSH trapping was performed as previously reported. ${ }^{1,2}$ In short, human liver microsomes (final concentration of $1 \mathrm{mg} / \mathrm{mL}$ ) in potassium phosphate buffer (100 mM, pH 7.4) was spiked with test compound (final concentration, $20 \mu \mathrm{M}$ ) fortified with $\mathrm{GSH}$ (final concentration of $1 \mathrm{mM}$ as a 1:1 mixture of stable labeled/unlabeled). The reaction was initiated by the addition of NADPH in buffer (final concentration of $1 \mathrm{mM}$ ). After 60 minute incubation at $37^{\circ} \mathrm{C}$, the reaction was quenched and microsomal proteins were precipitated by the addition of acetonitrile ( 3 fold incubation volume). After centrifugation at $2000 \mathrm{x} g$ for $10 \mathrm{~min}$, supernatants were removed and evaporated on Evaporex EVX-192 from Apricot Designs (Monrovia, CA) and reconstituted in 1:1 (v/v) of acetonitrile: water solution.

Aliquots of the samples were injected onto a Hypersil Gold $\mathrm{C}_{18}$ Column (1.9 micron, $2.1 \mathrm{x} 100$ $\mathrm{mm}$ ) from Thermo Scientific (San Jose, CA). Chromatographic separation was achieved using a solvent system consisting of mobile phase A (water containing $0.1 \%$ formic acid) and mobile phase B (ACN containing $0.1 \%$ formic acid) at a flow rate of $350 \mu \mathrm{L} / \mathrm{min}$. The LC/MS equipment consisted of an Accela solvent delivery system with built in degasser (Thermo Scientific) and an HTS PAL autosampler from CTC Analytics (Carrboro, NC), which were coupled to an LTQ-Orbitrap mass spectrometer from Thermo 
Scientific. The mass spectrometer was operated in the positive ion mode with data-dependent acquisition. Data-dependent acquisition was performed to acquire the full MS scans over a mass range of $\mathrm{m} / \mathrm{z} 200$ to 1000 followed by $\mathrm{MS}^{2}$ and $\mathrm{MS}^{3}$ scans with a collision energy of $17 \%$. MS ${ }^{2}$ was performed on the most intense peaks that had a mass difference of 3.0037 Da (the delta mass of both high and low masses of the labeled and unlabeled GSH) with a relative ratio of approximately $1 . \mathrm{MS}^{3}$ was triggered for the five most intense peaks that had a neutral loss of $129 \mathrm{Da}$ after GSH conjugate fragmentation.

For the fragments (MW <250 Da), peak heights in the full scan positive ion mode corresponding to GSH conjugates were measured in counts per second (cps). The measured cps values were summed for all conjugates to generate the total. These were categorized thusly: "+" for $10^{3}-10^{5}$; “++" for $10^{5}-10^{6}$; and "+++" for $>10^{6}$. Total amount of GSH conjugate formation was assessed, since some conjugates represent a combination of changes such as the loss of glutamic acid or oxidation.

For matched-pair drug discovery project compounds (MW > $250 \mathrm{Da}$ ), peak areas corresponding to all the conjugates were integrated and summed together. These values were normalized for differences in ionization efficiency through scaling by the measured ionization of a known concentration of parent molecule. For each set, the compound with the fewest ring nitrogens was normalized to $100 \%$, and all the others reported as a relative percentage of this value. 
Table S1: Peak height data for the bioactivation of fragments

\begin{tabular}{|c|c|c|}
\hline Entry & $\begin{array}{l}\text { Peak Height of GSH } \\
\text { Conjugates }\end{array}$ & $\begin{array}{c}\text { Relative intensity of } \\
\text { GSH conjugates }\end{array}$ \\
\hline 5 & 1.09.E+06 & +++ \\
\hline 6 & $0.00 . E+00$ & BLD \\
\hline 7 & $0.00 . E+00$ & BLD \\
\hline 8 & 1.78.E+06 & +++ \\
\hline 9 & $0.00 . E+00$ & BLD \\
\hline 10 & 2.15.E+05 & ++ \\
\hline 11 & 1.71.E+05 & ++ \\
\hline 12 & $2.75 . E+05$ & ++ \\
\hline 13 & $1.70 . \mathrm{E}+06$ & +++ \\
\hline 14 & 3.90.E+05 & ++ \\
\hline 15 & 1.24.E+06 & +++ \\
\hline 16 & $6.38 . E+04$ & + \\
\hline 17 & $0.00 . E+00$ & BLD \\
\hline 18 & $0.00 . E+00$ & BLD \\
\hline 19 & $0.00 . E+00$ & BLD \\
\hline 20 & $0.00 . E+00$ & BLD \\
\hline 21 & 4.04.E+05 & ++ \\
\hline 22 & 4.09.E+04 & + \\
\hline 23 & 3.90.E+04 & + \\
\hline 24 & $0.00 . E+00$ & BLD \\
\hline 25 & 4.29.E+04 & + \\
\hline 26 & 6.83.E+05 & ++ \\
\hline 27 & $0.00 . E+00$ & BLD \\
\hline 28 & $5.29 . E+05$ & ++ \\
\hline 29 & 2.48.E+05 & ++ \\
\hline 30 & 3.18.E+06 & +++ \\
\hline 31 & 7.99.E+05 & ++ \\
\hline 32 & $9.71 . E+06$ & +++ \\
\hline 33 & 2.30.E+06 & +++ \\
\hline 34 & 7.23.E+04 & + \\
\hline 35 & 4.68.E+04 & + \\
\hline 36 & $2.78 . E+04$ & + \\
\hline 37 & $0.00 . E+00$ & BLD \\
\hline 38 & $0.00 . E+00$ & BLD \\
\hline 39 & $0.00 . E+00$ & BLD \\
\hline 40 & $2.20 . E+04$ & + \\
\hline 41 & $0.00 . E+00$ & BLD \\
\hline 42 & 4.10.E+03 & + \\
\hline 43 & $8.80 . E+04$ & + \\
\hline 44 & $9.70 . \mathrm{E}+04$ & + \\
\hline 45 & $0.00 . E+00$ & BLD \\
\hline 46 & 3.13.E+05 & ++ \\
\hline 47 & $0.00 . E+00$ & BLD \\
\hline
\end{tabular}

BLD”, below limit of detection; “+” represents a range from $10^{3}-10^{5}$; “++”: $10^{5}-10^{6}$; “+++”: $>10^{6}$ cps. 
Table S2: MS data for parent molecules and their corresponding GSH-related conjugates

\begin{tabular}{|c|c|c|c|c|c|c|c|}
\hline \multirow[b]{2}{*}{ ID } & \multirow[b]{2}{*}{$m / z$} & \multicolumn{3}{|c|}{ Mass Spec Response of Parents } & \multicolumn{3}{|c|}{ Mass Spec Responses of GSH Conjugates } \\
\hline & & $\mathrm{A}$ & $\mathrm{B}$ & A/B & $\begin{array}{c}\text { Total GSH-related } \\
\text { Conjugates }\end{array}$ & $\begin{array}{c}\text { Ionization-adjusted } \\
\text { GSH Conjugates }\end{array}$ & $\begin{array}{l}\% \text { Adduct formation } \\
\text { relative to phenyl }\end{array}$ \\
\hline 48 & 298.2026 & $3.5 \mathrm{E}+08$ & $5.5 \mathrm{E}+07$ & 6.3 & $1.87 \mathrm{E}+06$ & $2.95 \mathrm{E}+05$ & 100 \\
\hline 49 & 299.1979 & $3.8 \mathrm{E}+08$ & $5.5 \mathrm{E}+07$ & 6.9 & $8.70 \mathrm{E}+04$ & $1.26 \mathrm{E}+04$ & 4 \\
\hline 50 & 300.1931 & $3.0 \mathrm{E}+08$ & $5.5 \mathrm{E}+07$ & 5.4 & BLD & BLD & BLD \\
\hline 51 & 486.2048 & $1.1 \mathrm{E}+09$ & $1.6 \mathrm{E}+07$ & 69.7 & $5.3 \mathrm{E}+07$ & $7.63 \mathrm{E}+05$ & 100 \\
\hline 52 & 487.2001 & $8.4 \mathrm{E}+08$ & $1.6 \mathrm{E}+07$ & 51.7 & $3.0 \mathrm{E}+07$ & $5.77 \mathrm{E}+05$ & 76 \\
\hline 53 & 343.1765 & $9.3 \mathrm{E}+07$ & $1.6 \mathrm{E}+07$ & 6.0 & $3.6 \mathrm{E}+06$ & $6.03 E+05$ & 100 \\
\hline 54 & 344.1717 & 1.3E+08 & 1.6E+07 & 8.2 & $7.6 \mathrm{E}+05$ & $9.24 E+04$ & 15 \\
\hline 55 & 450.1594 & $7.3 \mathrm{E}+07$ & $1.6 \mathrm{E}+07$ & 4.7 & $6.6 \mathrm{E}+07$ & $1.41 \mathrm{E}+07$ & 100 \\
\hline 56 & 451.1547 & $5.9 E+08$ & 1.6E+07 & 37.7 & 1.3E+08 & $3.57 E+06$ & 25 \\
\hline 57 & 470.1493 & $2.3 \mathrm{E}+07$ & 1.4E+07 & 1.7 & $2.9 E+06$ & $1.76 \mathrm{E}+06$ & 100 \\
\hline 58 & 471.1445 & $2.9 E+07$ & $1.4 \mathrm{E}+07$ & 2.0 & BLD & BLD & BLD \\
\hline 59 & 472.1398 & $2.5 \mathrm{E}+07$ & $1.4 \mathrm{E}+07$ & 1.8 & BLD & BLD & BLD \\
\hline 60 & 469.1540 & $4.9 E+07$ & $1.6 \mathrm{E}+07$ & 3.2 & $1.7 \mathrm{E}+07$ & $5.35 \mathrm{E}+06$ & 100 \\
\hline 61 & 471.1445 & $5.6 \mathrm{E}+07$ & $1.6 \mathrm{E}+07$ & 3.6 & $2.7 E+06$ & $7.33 \mathrm{E}+05$ & 14 \\
\hline 62 & 307.1441 & $1.5 \mathrm{E}+08$ & $1.1 \mathrm{E}+07$ & 13.4 & $9.9 \mathrm{E}+06$ & $7.38 \mathrm{E}+05$ & 100 \\
\hline 63 & 308.1394 & $9.7 E+07$ & $1.1 \mathrm{E}+07$ & 8.8 & $1.1 \mathrm{E}+07$ & $1.20 \mathrm{E}+06$ & 162 \\
\hline 64 & 308.1394 & 1.4E+09 & 1.4E+07 & 98.4 & $5.4 \mathrm{E}+07$ & $5.44 \mathrm{E}+05$ & 74 \\
\hline 65 & 611.3140 & $3.2 \mathrm{E}+07$ & $1.7 \mathrm{E}+07$ & 1.9 & $7.6 \mathrm{E}+07$ & $3.89 \mathrm{E}+07$ & 100 \\
\hline 66 & 612.3093 & $3.9 E+07$ & 1.7E+07 & 2.3 & $6.3 E+05$ & $2.72 E+05$ & 1 \\
\hline 67 & 613.3045 & $3.3 E+07$ & 1.7E+07 & 2.0 & BLD & BLD & BLD \\
\hline 68 & 627.2548 & $3.2 \mathrm{E}+07$ & $1.6 \mathrm{E}+07$ & 2.0 & $1.0 \mathrm{E}+07$ & $5.17 \mathrm{E}+06$ & 100 \\
\hline 69 & 628.2501 & $3.9 \mathrm{E}+07$ & $1.6 \mathrm{E}+07$ & 2.4 & $3.3 E+06$ & $1.37 E+06$ & 27 \\
\hline 70 & 629.2453 & $5.3 E+07$ & 1.6E+07 & 3.2 & BLD & BLD & BLD \\
\hline 71 & 654.3199 & $1.8 \mathrm{E}+08$ & $1.7 \mathrm{E}+07$ & 10.5 & $3.3 \mathrm{E}+06$ & $3.16 \mathrm{E}+05$ & 100 \\
\hline 72 & 655.3151 & $6.7 E+07$ & 1.7E+07 & 4.0 & $5.2 \mathrm{E}+05$ & $1.31 \mathrm{E}+05$ & 41 \\
\hline 73 & 655.3151 & $3.0 \mathrm{E}+08$ & 1.6E+07 & 18.4 & BLD & BLD & BLD \\
\hline 74 & 457.1305 & $1.2 \mathrm{E}+09$ & $1.5 \mathrm{E}+07$ & 76.7 & $1.7 \mathrm{E}+06$ & $2.16 \mathrm{E}+04$ & 100 \\
\hline 75 & 458.1257 & 1.2E+09 & $1.5 \mathrm{E}+07$ & 81.2 & $1.5 \mathrm{E}+06$ & $1.84 E+04$ & 85 \\
\hline
\end{tabular}

A: Peak area of parent on mass spec

B: Peak area of internal standard (propranolol) on mass spec

$\mathrm{BLD}=$ Below Limit of Detection 


\section{Chemical Characterization and Purity}

Purity of samples was assessed by LC and compounds were characterized by MS (see listed table below). Purity of fragments by LC-MS was $>90 \%$ with the exception of compounds $\mathbf{1 0}$ and $\mathbf{4 7}$. For project compounds, purity by LC-MS was $\geq 95 \%$ with the exception of compound $\mathbf{5 4}$. Owing to the large dataset, the historical nature of many project samples, and limitations in available solid stock, ${ }^{1} \mathrm{H}-\mathrm{NMR}$ spectra were not collected for this compound set. Compound $\mathbf{4 7}$ was unstable to LC/MS conditions, and purity was assessed and confirmed by ${ }^{1} \mathrm{H}-\mathrm{NMR}$.

\section{LCMS method A: Agilent 10-min}

Experiments performed on an Agilent 1290 UHPLC coupled with Agilent MSD (6140) mass spectrometer using ESI as ionization source. The LC separation was using a Phenomenex XB-C18, 1.7um, $50 \times 2.1 \mathrm{~mm}$ column with a $0.4 \mathrm{~mL} /$ minute flow rate. Solvent $\mathrm{A}$ is water with $0.1 \% \mathrm{FA}$ and solvent B is acetonitrile with $0.1 \%$ FA. The gradient consisted with $2-98 \%$ solvent B over 7 min and hold $98 \%$ B for $1.5 \mathrm{~min}$ following equilibration for $1.5 \mathrm{~min}$. LC column temperature is $40{ }^{\circ} \mathrm{C}$. UV absorbance was collected at $220 \mathrm{~nm}$ and $254 \mathrm{~nm}$ and mass spec full scan was applied to all experiment.

\section{LCMS method B: Waters 20-min}

Experiment was performed on a Waters Acquity UHPLC with Waters LCT Premier XE mass spectrometer using ESI ionization. The HPLC method was a gradient flow of $0.6 \mathrm{~mL} / \mathrm{min}$ starting from $98 \%$ water with $0.05 \%$ TFA (solvent A) and $2 \%$ acetonitrile with $0.05 \%$ TFA (solvent B), ramping up to 2\% solvent A and 98\% solvent B over 17.5 minutes. The final solvent system was held constant for a further 0.5 minute. LC column was Waters Acquity UPLC BEH C18, 1.7um, 2.1×50mm with column temperature of $40{ }^{\circ} \mathrm{C}$. UV absorbance was collected at $220 \mathrm{~nm}$ and $254 \mathrm{~nm}$ and mass spec full scan was acquired.

\section{LCMS method C: Waters 3-min}


Experiment was performed on a Waters Acquity UHPLC with Waters LCT Premier XE mass spectrometer using ESI ionization. The HPLC method was a gradient flow of $0.6 \mathrm{~mL} / \mathrm{min}$ starting from $98 \%$ water with $0.05 \%$ TFA (solvent A) and $2 \%$ acetonitrile with $0.05 \%$ TFA (solvent B), ramping up to $2 \%$ solvent A and $98 \%$ solvent B over 2.5 minutes. The final solvent system was held constant for a further 0.5 minute. LC column was Waters Acquity UPLC BEH C18, 1.7um, 2.1×50mm with column temperature of $40{ }^{\circ} \mathrm{C}$. UV absorbance was collected at $220 \mathrm{~nm}$ and $254 \mathrm{~nm}$ and mass spec full scan was acquired.

\section{LCMS method D: Waters 6-min}

Experiments performed on a Waters Acquity UPLC with Waters LCT Premier XE mass spectrometer using ESI as ionization source. The LC separation was using an Acquity UPLC BEH C18, 1.7um, $2.1 \times 50 \mathrm{~mm}$ column and a $0.6 \mathrm{ml} /$ minute flow rate. Solvent A is water with $0.05 \% \mathrm{TFA}$ and solvent $\mathrm{B}$ is acetonitrile with $0.05 \%$ TFA. The gradient consisted with $2-98 \%$ solvent B over 5 min and hold $98 \% \mathrm{~B}$ for $0.5 \mathrm{~min}$ following equilibration for $0.5 \mathrm{~min}$. LC column temperature is $40{ }^{\circ} \mathrm{C}$. UV absorbance was collected at 210-254nm and mass spec full scan was applied to all experiment.

Table S3: Purity and characterization for all compounds by LC-MS

\begin{tabular}{|l|l|l|l|l|}
\hline $\begin{array}{c}\text { Entry } \\
\text { Number }\end{array}$ & \multicolumn{1}{|c|}{ IUPAC } & $\begin{array}{c}\text { LCMS } \\
(\mathrm{ESI}), \mathrm{m} / \mathrm{z} \\
{[\mathrm{M}+\mathrm{H}]^{+}}\end{array}$ & $\begin{array}{c}\text { Compound \% Purity } \\
\text { by LC-MS (uv = 254 } \\
\mathrm{nM} \text { ) }\end{array}$ & $\begin{array}{l}\text { LC-MS } \\
\text { Method }\end{array}$ \\
\hline 5 & $N^{1}$-methylbenzene-1,4-diamine & 123.1 & 100 & $\mathrm{~A}$ \\
\hline 6 & $N^{2}$-methylpyridine-2,5-diamine & 124.1 & 100 & $\mathrm{~A}$ \\
\hline 7 & $N^{3}$-methylpyridazine-3,6-diamine & 125.1 & 100 & $\mathrm{~A}$ \\
\hline 8 & $\begin{array}{l}N^{1} \text {-methyl-3-(trifluoromethyl)benzene- } \\
\text { 1,4-diamine }\end{array}$ & 191.0 & 96.5 & $\mathrm{~A}$ \\
\hline 9 & 2-fluoro- $N^{1}$-methylbenzene-1,4-diamine & 141.1 & 98 & $\mathrm{~A}$ \\
\hline 10 & $\begin{array}{l}\text { 2-chloro- } N^{1} \text {-methylbenzene-1,4- } \\
\text { diamine }\end{array}$ & 157.1 & 88.7 & $\mathrm{~A}$ \\
\hline 11 & $\begin{array}{l}\text { 2-bromo- } N^{1} \text {-methylbenzene-1,4- } \\
\text { diamine }\end{array}$ & 201.9 & 90.5 & $\mathrm{~A}$ \\
\hline 12 & 5-amino-2-(methylamino)benzonitrile & 148.1 & 100 & $\mathrm{~A}$ \\
\hline 13 & 4-aminophenol & 110.1 & 100 & $\mathrm{D}$ \\
\hline 14 & 4-(methylamino)phenol & 124.1 & 100 & $\mathrm{~A}$ \\
\hline 15 & 4-morpholinophenol & 180.1 & 94 & $\mathrm{~A}$ \\
\hline 16 & 2-(methylamino)pyrimidin-5-ol & 126.1 & 96.6 & $\mathrm{D}$ \\
\hline
\end{tabular}




\begin{tabular}{|c|c|c|c|c|}
\hline 17 & 6-aminopyridin-3-ol & 111.1 & 94 & $\mathrm{~A}$ \\
\hline 18 & 6-aminopyridazin-3-ol & 112.1 & 100 & A \\
\hline 19 & 6-(methylamino)pyridazin-3-ol & 126.1 & 96.7 & $\mathrm{~A}$ \\
\hline 20 & $\begin{array}{l}\text { 6-(4-methylpiperazin-1-yl)pyridazin-3- } \\
\text { ol }\end{array}$ & 195.1 & 100 & $\mathrm{~A}$ \\
\hline 21 & 4-morpholinoaniline & 179.1 & 100 & $\mathrm{~A}$ \\
\hline 22 & 5-morpholinopyrazin-2-amine & 181.1 & 90 & $\mathrm{~A}$ \\
\hline 23 & 6-morpholinopyridin-3-amine & 180.1 & 100 & $\mathrm{~A}$ \\
\hline 24 & 5-morpholinopyridin-2-amine & 180.1 & 100 & $\mathrm{~A}$ \\
\hline 25 & 5-morpholinopyrimidin-2-amine & 181.2 & 99.4 & $\mathrm{~A}$ \\
\hline 26 & 2-morpholinopyrimidin-5-amine & 181.1 & 98.9 & $\mathrm{~A}$ \\
\hline 27 & 6-morpholinopyridazin-3-amine & 181.2 & 100 & $\mathrm{~A}$ \\
\hline 28 & 2-methyl-4-morpholinoaniline & 193.1 & 100 & $\mathrm{D}$ \\
\hline 29 & 4-morpholino-2-(trifluoromethyl)aniline & 247.1 & 100 & $\mathrm{C}$ \\
\hline 30 & 4-morpholino-3-(trifluoromethyl)aniline & 247.1 & 100 & $\mathrm{C}$ \\
\hline 31 & 3-chloro-4-morpholinoaniline & 213.0 & 99.4 & $\mathrm{~A}$ \\
\hline 32 & 3-fluoro-4-morpholinoaniline & 197.1 & 95 & $\mathrm{~A}$ \\
\hline 33 & 3-bromo-4-morpholinoaniline & 257.1 & 99 & $\mathrm{~A}$ \\
\hline 34 & 3-methyl-4-morpholinoaniline & 193.1 & 99.3 & $\mathrm{~A}$ \\
\hline 35 & 4-morpholinobenzene-1,3-diamine & 194.1 & 100 & $\mathrm{C}$ \\
\hline 36 & 4-(4-methylpiperazin-1-yl)aniline & 192.1 & 100 & $\mathrm{D}$ \\
\hline 37 & $\begin{array}{l}\text { 5-(4-methylpiperazin-1-yl)pyrazin-2- } \\
\text { amine }\end{array}$ & 194.1 & 100 & $\mathrm{~A}$ \\
\hline 38 & $\begin{array}{l}\text { 6-(4-methylpiperazin-1-yl)pyridin-3- } \\
\text { amine }\end{array}$ & 193.1 & 100 & $\mathrm{D}$ \\
\hline 39 & $\begin{array}{l}\text { 5-(4-methylpiperazin-1-yl)pyridin-2- } \\
\text { amine }\end{array}$ & 193.1 & 100 & $\mathrm{~A}$ \\
\hline 40 & $\begin{array}{l}\text { 2-(4-methylpiperazin-1-yl)pyrimidin-5- } \\
\text { amine }\end{array}$ & 194.1 & 100 & $\bar{A}$ \\
\hline 41 & $\begin{array}{l}\text { 6-(4-methylpiperazin-1-yl)pyridazin-3- } \\
\text { amine }\end{array}$ & 194.1 & 100 & A \\
\hline 42 & $\begin{array}{l}\text { 2-methyl-4-(4-methylpiperazin-1- } \\
\text { yl)aniline }\end{array}$ & 206.1 & 100 & $\mathrm{~A}$ \\
\hline 43 & $\begin{array}{l}\text { 4-(4-methylpiperazin-1-yl)-2- } \\
\text { (trifluoromethyl)aniline }\end{array}$ & 260.1 & 99.9 & A \\
\hline 44 & $\begin{array}{l}\text { 4-(4-methylpiperazin-1-yl)-3- } \\
\text { (trifluoromethyl)aniline }\end{array}$ & 260.1 & 100 & $\mathrm{~A}$ \\
\hline 45 & $\begin{array}{l}\text { 3-chloro-4-(4-methylpiperazin-1- } \\
\text { yl)aniline }\end{array}$ & 226.1 & 100 & $\mathrm{D}$ \\
\hline 46 & $\begin{array}{l}\text { 3-fluoro-4-(4-methylpiperazin-1- } \\
\text { yl)aniline }\end{array}$ & 210.1 & 100 & $\mathrm{D}$ \\
\hline 47 & $\begin{array}{l}\text { 4-(4-methylpiperazin-1-yl)benzene-1,2- } \\
\text { diamine }\end{array}$ & 207.1 & $\begin{array}{l}\text { 63* (unstable on LC- } \\
\text { MS; purity by NMR) }\end{array}$ & $\bar{A}$ \\
\hline 48 & $\begin{array}{l}\text { 5-cyclopropyl- } N \text {-(4-(4-methylpiperazin- } \\
\text { 1-yl)phenyl)- } 1 H \text {-pyrazol-3-amine }\end{array}$ & 298.2 & 94 & $\mathrm{D}$ \\
\hline 49 & $\begin{array}{l}N \text {-(5-cyclopropyl-1 } H \text {-pyrazol-3-yl)-5- } \\
\text { (4-methylpiperazin-1-yl)pyridin-2- } \\
\text { amine }\end{array}$ & 299.1 & 100 & $\mathrm{D}$ \\
\hline
\end{tabular}




\begin{tabular}{|c|c|c|c|c|}
\hline 50 & $\begin{array}{l}N \text {-(5-cyclopropyl-1H-pyrazol-3-yl)-6- } \\
\text { (4-methylpiperazin-1-yl)pyridazin-3- } \\
\text { amine }\end{array}$ & 300.1 & 100 & $\mathrm{D}$ \\
\hline 51 & $\begin{array}{l}\text { 4-((5-fluoro-2-((4- } \\
\text { morpholinophenyl)amino)pyrimidin-4- } \\
\text { yl)amino)-N-(pyridin-2-yl)benzamide }\end{array}$ & 486.2 & 98 & A \\
\hline 52 & $\begin{array}{l}\text { 4-((5-fluoro-2-((6-morpholinopyridin-3- } \\
\text { yl)amino)pyrimidin-4-yl)amino)-N- } \\
\text { (pyridin-2-yl)benzamide }\end{array}$ & 487.2 & 100 & A \\
\hline 53 & $\begin{array}{l}\text { 1-ethyl-3-(4-(5-morpholino-2- } \\
\text { oxopyridin-1(2H)-yl)phenyl)urea }\end{array}$ & 343.2 & 100 & A \\
\hline 54 & $\begin{array}{l}\text { 1-ethyl-3-(5-morpholino-2-oxo-2H- } \\
\text { [1,2'-bipyridin]-5'-yl)urea }\end{array}$ & 344.1 & 93.9 & $\mathrm{~A}$ \\
\hline 55 & $\begin{array}{l}\text { 5-(3-(methylsulfonyl)phenyl)-N-(4- } \\
\text { morpholinophenyl)-[1,2,4]triazolo[1,5- } \\
\text { a]pyridin-2-amine }\end{array}$ & 450.1 & 100 & D \\
\hline 56 & $\begin{array}{l}\text { 5-(3-(methylsulfonyl)phenyl)-N-(6- } \\
\text { morpholinopyridin-3-yl)- } \\
{[1,2,4] \text { triazolo[1,5-a]pyridin-2-amine }}\end{array}$ & 451.1 & 95 & D \\
\hline 57 & $\begin{array}{l}\mathrm{N}-(4-(1,1- \\
\text { dioxidothiomorpholino)phenyl)-4-((2- } \\
\text { methoxyphenyl)amino)-6-oxo-1,6- } \\
\text { dihydropyrimidine-5-carboxamide }\end{array}$ & 470.1 & 100 & $\mathrm{D}$ \\
\hline 58 & $\begin{array}{l}\text { N-(6-(1,1- } \\
\text { dioxidothiomorpholino)pyridin-3-yl)-4- } \\
\text { ((2-methoxyphenyl)amino)-6-oxo-1,6- } \\
\text { dihydropyrimidine-5-carboxamide }\end{array}$ & 471.1 & 100 & $\mathrm{D}$ \\
\hline 59 & $\begin{array}{l}\mathrm{N}-(2-(1,1- \\
\text { dioxidothiomorpholino)pyrimidin-5-yl)- } \\
\text { 4-((2-methoxyphenyl)amino)-6-oxo- } \\
\text { 1,6-dihydropyrimidine-5-carboxamide }\end{array}$ & 472.1 & 100 & $\mathrm{D}$ \\
\hline 60 & $\begin{array}{l}\mathrm{N}-(4-(1,1- \\
\text { dioxidothiomorpholino)phenyl)-4-((2- } \\
\text { methoxyphenyl)amino)-2-oxo-1,2- } \\
\text { dihydropyridine-3-carboxamide }\end{array}$ & 469.2 & 100 & $A$ \\
\hline 61 & $\begin{array}{l}\mathrm{N}-(2-(1,1- \\
\text { dioxidothiomorpholino)pyrimidin-5-yl)- } \\
\text { 4-((2-methoxyphenyl)amino)-2-oxo- } \\
\text { 1,2-dihydropyridine-3-carboxamide }\end{array}$ & 471.1 & 100 & $\mathrm{D}$ \\
\hline 62 & $\begin{array}{l}\text { N-(4-(pyrrolidin-1- } \\
\text { yl)phenyl)benzofuran-2-carboxamide }\end{array}$ & 307.1 & 100 & $\mathrm{C}$ \\
\hline 63 & $\begin{array}{l}\text { N-(5-(pyrrolidin-1-yl)pyridin-2- } \\
\text { yl)benzofuran-2-carboxamide }\end{array}$ & 308.1 & 100 & A \\
\hline 64 & $\begin{array}{l}\text { N-(6-(pyrrolidin-1-yl)pyridin-3- } \\
\text { yl)benzofuran-2-carboxamide }\end{array}$ & 308.1 & 97.8 & A \\
\hline 65 & $\begin{array}{l}\text { 2-(5-fluoro-2-(hydroxymethyl)-3-(1- } \\
\text { methyl-5-((4-(4-methylpiperazin-1- } \\
\text { yl)phenyl)amino)-6-oxo-1,6- } \\
\text { dihydropyridin-3-yl)phenyl)- }\end{array}$ & 611.2 & 97.3 & A \\
\hline
\end{tabular}




\begin{tabular}{|c|c|c|c|c|}
\hline & $\begin{array}{l}\text { 3,4,6,7,8,9-hexahydropyrazino[1,2- } \\
\text { a]indol-1(2H)-one }\end{array}$ & & & \\
\hline 66 & $\begin{array}{l}\text { 2-(5-fluoro-2-(hydroxymethyl)-3-(1- } \\
\text { methyl-5-((5-(4-methylpiperazin-1- } \\
\text { yl)pyridin-2-yl)amino)-6-oxo-1,6- } \\
\text { dihydropyridin-3-yl)phenyl)- } \\
\text { 3,4,6,7,8,9-hexahydropyrazino[1,2- } \\
\text { a]indol-1(2H)-one }\end{array}$ & 612.3 & 100 & B \\
\hline 67 & $\begin{array}{l}\text { 2-(5-fluoro-2-(hydroxymethyl)-3-(1- } \\
\text { methyl-5-((6-(4-methylpiperazin-1- } \\
\text { yl)pyridazin-3-yl)amino)-6-oxo-1,6- } \\
\text { dihydropyridin-3-yl)phenyl)- } \\
\text { 3,4,6,7,8,9-hexahydropyrazino[1,2- } \\
\text { a]indol-1(2H)-one }\end{array}$ & 613.1 & 100 & $\mathrm{D}$ \\
\hline 68 & $\begin{array}{l}\text { 3-(5-fluoro-2-(hydroxymethyl)-3-(1- } \\
\text { methyl-5-((4-(4-methylpiperazin-1- } \\
\text { yl)phenyl)amino)-6-oxo-1,6- } \\
\text { dihydropyridin-3-yl)phenyl)-6,7,8,9- } \\
\text { tetrahydrobenzo[4,5]thieno[2,3- } \\
\text { d]pyridazin-4(3H)-one }\end{array}$ & 627.2 & 100 & $\mathrm{~A}$ \\
\hline 69 & $\begin{array}{l}\text { 3-(5-fluoro-2-(hydroxymethyl)-3-(1- } \\
\text { methyl-5-((5-(4-methylpiperazin-1- } \\
\text { yl)pyridin-2-yl)amino)-6-oxo-1,6- } \\
\text { dihydropyridin-3-yl)phenyl)-6,7,8,9- } \\
\text { tetrahydrobenzo[4,5]thieno[2,3- } \\
\text { d]pyridazin-4(3H)-one }\end{array}$ & 628.2 & 100 & B \\
\hline 70 & $\begin{array}{l}\text { 3-(5-fluoro-2-(hydroxymethyl)-3-(1- } \\
\text { methyl-5-((6-(4-methylpiperazin-1- } \\
\text { yl)pyridazin-3-yl)amino)-6-oxo-1,6- } \\
\text { dihydropyridin-3-yl)phenyl)-6,7,8,9- } \\
\text { tetrahydrobenzo[4,5]thieno[2,3- } \\
\text { d]pyridazin-4(3H)-one }\end{array}$ & 629.1 & 100 & $\mathrm{D}$ \\
\hline 71 & $\begin{array}{l}\text { 2-(5-fluoro-2-(hydroxymethyl)-3-(1- } \\
\text { methyl-5-((5-(4-(oxetan-3-yl)piperazin- } \\
\text { 1-yl)pyridin-2-yl)amino)-6-oxo-1,6- } \\
\text { dihydropyridin-3-yl)phenyl)- } \\
\text { 3,4,6,7,8,9-hexahydropyrazino[1,2- } \\
\text { a]indol-1(2H)-one }\end{array}$ & 654.4 & 100 & B \\
\hline 72 & $\begin{array}{l}\text { 2-(5-fluoro-2-(hydroxymethyl)-3-(1- } \\
\text { methyl-5-((5-(4-(oxetan-3-yl)piperazin- } \\
\text { 1-yl)pyrimidin-2-yl)amino)-6-oxo-1,6- } \\
\text { dihydropyridin-3-yl)phenyl)- } \\
\text { 3,4,6,7,8,9-hexahydropyrazino[1,2- } \\
\text { a]indol-1(2H)-one }\end{array}$ & 655.2 & 100 & $\mathrm{D}$ \\
\hline 73 & $\begin{array}{l}\text { 2-(5-fluoro-2-(hydroxymethyl)-3-(1- } \\
\text { methyl-5-((6-(4-(oxetan-3-yl)piperazin- } \\
\text { 1-yl)pyridazin-3-yl)amino)-6-oxo-1,6- } \\
\text { dihydropyridin-3-yl)phenyl)- } \\
\text { 3,4,6,7,8,9-hexahydropyrazino[1,2- } \\
\text { a]indol-1(2H)-one }\end{array}$ & 655.2 & 100 & B \\
\hline
\end{tabular}




\begin{tabular}{|l|l|l|l|l|}
\hline 74 & $\begin{array}{l}\text { 2,6-dichloro-N-(2-((5-(4- } \\
\text { methylpiperazin-1-yl)pyridin-2- } \\
\text { yl)amino)pyridin-4-yl)benzamide }\end{array}$ & 457.2 & 96.6 & $\mathrm{~A}$ \\
\hline 75 & $\begin{array}{l}\text { 2,6-dichloro-N-(2-((6-(4- } \\
\text { methylpiperazin-1-yl)pyridazin-3- } \\
\text { yl)amino)pyridin-4-yl)benzamide }\end{array}$ & 458.1 & 100 & $\mathrm{~A}$ \\
\hline
\end{tabular}

*Not stable in LC-MS; NMR data: ${ }^{1} \mathrm{H}$ NMR $\left(400 \mathrm{MHz}, \mathrm{DMSO}-d_{6}\right) \delta 6.39(\mathrm{~d}, \mathrm{~J}=8.3 \mathrm{~Hz}, 1 \mathrm{H}), 6.21(\mathrm{~d}, \mathrm{~J}=$ $2.7 \mathrm{~Hz}, 1 \mathrm{H}), 6.01(\mathrm{dd}, \mathrm{J}=8.4,2.7 \mathrm{~Hz}, 1 \mathrm{H}), 4.33(\mathrm{~s}, 2 \mathrm{H}), 3.06(\mathrm{~s}, 2 \mathrm{H}), 2.90-2.83(\mathrm{~m}, 4 \mathrm{H}), 2.43-2.36(\mathrm{~m}$, $4 \mathrm{H}), 2.19(\mathrm{~s}, 3 \mathrm{H})$.

\section{H-NMR and HRMS characterization of compounds 48-75}

48: ${ }^{1} \mathrm{H}$ NMR (400 MHz, DMSO- $\left.d_{6}\right) \delta 11.50(\mathrm{~s}, 1 \mathrm{H}), 7.81(\mathrm{~s}, 1 \mathrm{H}), 7.15(\mathrm{~s}, 2 \mathrm{H}), 6.83-6.74(\mathrm{~m}, 2 \mathrm{H}), 5.41$ $(\mathrm{s}, 1 \mathrm{H}), 2.96(\mathrm{t}, \mathrm{J}=5.0 \mathrm{~Hz}, 4 \mathrm{H}), 2.43(\mathrm{t}, \mathrm{J}=5.0 \mathrm{~Hz}, 4 \mathrm{H}), 2.21(\mathrm{~s}, 3 \mathrm{H}), 1.88-1.76(\mathrm{~m}, 1 \mathrm{H}), 0.91-0.84$ $(\mathrm{m}, 2 \mathrm{H}), 0.67-0.61(\mathrm{~m}, 2 \mathrm{H})$. HRMS (ESI-) $\mathrm{m} / \mathrm{z}$ found $[\mathrm{M}-\mathrm{H}]^{-} 296.1873, \mathrm{C}_{17} \mathrm{H}_{22} \mathrm{~N}_{5}$ requires 296.1881.

49: ${ }^{1} \mathrm{H}$ NMR (400 MHz, DMSO- $\left.d_{6}\right) \delta 11.46(\mathrm{~s}, 1 \mathrm{H}), 8.17(\mathrm{~s}, 1 \mathrm{H}), 7.81-7.76(\mathrm{~m}, 1 \mathrm{H}), 7.28$ - $7.20(\mathrm{~m}$, $2 \mathrm{H}), 5.81(\mathrm{~s}, 1 \mathrm{H}), 3.04-2.97(\mathrm{~m}, 4 \mathrm{H}), 2.49-2.42(\mathrm{~m}, 4 \mathrm{H}), 2.22(\mathrm{~s}, 3 \mathrm{H}), 1.88-1.74(\mathrm{~m}, 1 \mathrm{H}), 0.89-0.82$ $(\mathrm{m}, 2 \mathrm{H}), 0.68-0.59(\mathrm{~m}, 2 \mathrm{H}) .{ }^{13} \mathrm{C}$ NMR $(101 \mathrm{MHz}, \mathrm{DMSO}) \delta 158.50,158.20,150.41,139.37,136.08$, $128.27,110.06,90.62,53.72,48.36,43.96,8.13,7.63$.

50: ${ }^{1} \mathrm{H}$ NMR (400 MHz, DMSO-d $\left.d_{6}\right) 11.48(\mathrm{~s}, 1 \mathrm{H}), 8.54(\mathrm{~s}, 1 \mathrm{H}), 7.27(\mathrm{~d}, \mathrm{~J}=9.5 \mathrm{~Hz}, 1 \mathrm{H}), 7.08(\mathrm{~d}, \mathrm{~J}=9.7$ $\mathrm{Hz}, 1 \mathrm{H}), 5.82(\mathrm{~s}, 1 \mathrm{H}), 3.35-3.28(\mathrm{~m}, 4 \mathrm{H}), 2.39-2.32(\mathrm{~m}, 4 \mathrm{H}), 2.15(\mathrm{~s}, 3 \mathrm{H}), 1.82-1.72(\mathrm{~m}, 1 \mathrm{H}), 0.85-$ $0.72(\mathrm{~m}, 2 \mathrm{H}), 0.63-0.52(\mathrm{~m}, 2 \mathrm{H}) .{ }^{13} \mathrm{C}$ NMR (101 MHz, DMSO) $\delta 156.67,152.60,152.48,118.54$, 117.42, 91.52, 54.86, 46.44, 46.21, 7.80. HRMS (ESI+) $\mathrm{m} / \mathrm{z}$ found $[\mathrm{M}+\mathrm{H}]^{+} 300.1934, \mathrm{C}_{15} \mathrm{H}_{22} \mathrm{~N}_{7}$ requires 300.1931 .

51: no sample available.

52: ${ }^{1} \mathrm{H}$ NMR (400 MHz, DMSO- $\left.d_{6}\right) \delta 10.71(\mathrm{~s}, 1 \mathrm{H}), 9.88-9.79(\mathrm{~m}, 1 \mathrm{H}), 9.47(\mathrm{~s}, 1 \mathrm{H}), 8.47-8.37(\mathrm{~m}$, $2 \mathrm{H}), 8.21(\mathrm{~d}, \mathrm{~J}=3.8 \mathrm{~Hz}, 1 \mathrm{H}), 8.19-8.15(\mathrm{~m}, 1 \mathrm{H}), 8.06-7.99(\mathrm{~m}, 3 \mathrm{H}), 7.95(\mathrm{~d}, \mathrm{~J}=8.6 \mathrm{~Hz}, 2 \mathrm{H}), 7.91-$ $7.85(\mathrm{~m}, 1 \mathrm{H}), 7.23-7.15(\mathrm{~m}, 2 \mathrm{H}), 3.76-3.71(\mathrm{~m}, 4 \mathrm{H}), 3.51-3.46(\mathrm{~m}, 4 \mathrm{H}) .{ }^{13} \mathrm{C} \mathrm{NMR}(101 \mathrm{MHz}$, DMSO) $\delta 165.46,158.36,158.00,154.89,152.02,149.78$ (d), 147.28, 142.33, 141.98, 139.50, 138.66, 134.67, 128.77, 128.42, 127.97, 119.94, 119.73, 114.79, 109.95, 65.52, 45.76. HRMS (ESI+) m/z found $[\mathrm{M}+\mathrm{H}]^{+} 487.1995, \mathrm{C}_{25} \mathrm{H}_{24} \mathrm{FN}_{8} \mathrm{O}_{2}$ requires 487.2006.

53: HRMS (ESI+) m/z found $[\mathrm{M}+\mathrm{H}]^{+} 343.1773, \mathrm{C}_{18} \mathrm{H}_{23} \mathrm{~N}_{4} \mathrm{O}_{3}$ requires 343.1765.

54: ${ }^{1} \mathrm{H}$ NMR (400 MHz, DMSO- $\left.d_{6}\right) \delta 8.81(\mathrm{~s}, 1 \mathrm{H}), 8.55(\mathrm{~d}, \mathrm{~J}=2.5 \mathrm{~Hz}, 1 \mathrm{H}), 7.97(\mathrm{dd}, \mathrm{J}=8.8,2.7 \mathrm{~Hz}$, $1 \mathrm{H}), 7.63(\mathrm{~d}, \mathrm{~J}=8.8 \mathrm{~Hz}, 1 \mathrm{H}), 7.57(\mathrm{dd}, \mathrm{J}=9.9,3.2 \mathrm{~Hz}, 1 \mathrm{H}), 7.17(\mathrm{~d}, \mathrm{~J}=3.0 \mathrm{~Hz}, 1 \mathrm{H}), 6.47(\mathrm{~d}, \mathrm{~J}=9.9 \mathrm{~Hz}$, $1 \mathrm{H}), 6.31(\mathrm{t}, \mathrm{J}=5.6 \mathrm{~Hz}, 1 \mathrm{H}), 3.96-3.48(\mathrm{~m}, 4 \mathrm{H}), 3.19-3.10(\mathrm{~m}, 2 \mathrm{H}), 3.00-2.70(\mathrm{~m}, 4 \mathrm{H}), 1.07(\mathrm{t}, \mathrm{J}=$ $7.2 \mathrm{~Hz}, 3 \mathrm{H}) .{ }^{13} \mathrm{C}$ NMR (101 MHz, DMSO) $\delta 159.22,154.90,145.24,137.66,136.92,136.82,133.10$, 126.16, 125.98, 121.28, 120.97, 117.35, 65.98, 50.14. HRMS (ESI+) $\mathrm{m} / \mathrm{z}$ found $[\mathrm{M}+\mathrm{H}]^{+} 344.1717$, $\mathrm{C}_{17} \mathrm{H}_{22} \mathrm{~N}_{5} \mathrm{O}_{3}$ requires 344.1717 .

55: ${ }^{1} \mathrm{H}$ NMR $\left(400 \mathrm{MHz}, \mathrm{DMSO}-d_{6}\right) \delta 9.38(\mathrm{~s}, 1 \mathrm{H}), 8.78-8.74(\mathrm{~m}, 1 \mathrm{H}), 8.38-8.33(\mathrm{~m}, 1 \mathrm{H}), 8.13-8.08$ $(\mathrm{m}, 1 \mathrm{H}), 7.88(\mathrm{t}, \mathrm{J}=7.8 \mathrm{~Hz}, 1 \mathrm{H}), 7.67(\mathrm{dd}, \mathrm{J}=8.8,7.2 \mathrm{~Hz}, 1 \mathrm{H}), 7.60(\mathrm{dd}, \mathrm{J}=8.7,1.4 \mathrm{~Hz}, 1 \mathrm{H}), 7.58-7.53$ $(\mathrm{m}, 2 \mathrm{H}), 7.32(\mathrm{dd}, \mathrm{J}=7.1,1.4 \mathrm{~Hz}, 1 \mathrm{H}), 6.92-6.84(\mathrm{~m}, 2 \mathrm{H}), 3.76-3.70(\mathrm{~m}, 4 \mathrm{H}), 3.01-2.96(\mathrm{dd}, \mathrm{J}=5.8$, $3.8 \mathrm{~Hz}, 4 \mathrm{H}), 1.23$ (s, 3H). ${ }^{13} \mathrm{C}$ NMR (101 MHz, DMSO) $\delta 162.19,150.49,145.17,141.03,134.13$, 
136.99, 133.81, 133.33, 129.82 (d), 128.01, 127.47, 117.63, 116.29, 112.69 (d), 66.19, 49.61, 43.54 . HRMS (ESI+) $\mathrm{m} / \mathrm{z}$ found $[\mathrm{M}-\mathrm{H}]^{-} 448.1440, \mathrm{C}_{23} \mathrm{H}_{22} \mathrm{~N}_{5} \mathrm{O}_{3} \mathrm{~S}$ requires 448.1449.

56: ${ }^{1} \mathrm{H}$ NMR (400 MHz, DMSO- $\left.d_{6}\right) \delta 10.06(\mathrm{~s}, 1 \mathrm{H}), 8.54(\mathrm{~d}, \mathrm{~J}=2.1 \mathrm{~Hz}, 1 \mathrm{H}), 8.52(\mathrm{~d}, \mathrm{~J}=2.7 \mathrm{~Hz}, 1 \mathrm{H})$, $8.46-8.39(\mathrm{~m}, 1 \mathrm{H}), 8.23(\mathrm{dd}, \mathrm{J}=9.6,2.8 \mathrm{~Hz}, 1 \mathrm{H}), 8.11(\mathrm{dd}, \mathrm{J}=7.8,1.5 \mathrm{~Hz}, 1 \mathrm{H}), 7.96(\mathrm{t}, \mathrm{J}=7.8 \mathrm{~Hz}, 1 \mathrm{H})$, $7.79-7.67(\mathrm{~m}, 2 \mathrm{H}), 7.41(\mathrm{dd}, \mathrm{J}=8.2,5.3 \mathrm{~Hz}, 2 \mathrm{H}), 3.77(\mathrm{~m}, 4 \mathrm{H}), 3.62(\mathrm{t}, \mathrm{J}=4.8 \mathrm{~Hz}, 4 \mathrm{H}), 3.34(\mathrm{~s}, 3 \mathrm{H})$. ${ }^{13} \mathrm{C}$ NMR (101 MHz, DMSO) $\delta 161.04,150.57,148.71,141.19,137.42,133.86,133.10,130.53,129.97$, 129.93, 128.05, 127.38, 113.69, 113.39, 112.97, 65.24, 63.19, 46.20, 43.45, 42.63. HRMS (ESI+) m/z found $[\mathrm{M}+\mathrm{H}]^{+} 451.1542, \mathrm{C}_{22} \mathrm{H}_{23} \mathrm{~N}_{6} \mathrm{O}_{3} \mathrm{~S}$ requires 451.1552 .

57: ${ }^{1} \mathrm{H}$ NMR (400 MHz, DMSO- $\left.d_{6}\right) \delta 12.62$ (br s, $\left.1 \mathrm{H}\right), 12.56(\mathrm{~s}, 1 \mathrm{H}), 12.20(\mathrm{~s}, 1 \mathrm{H}), 8.18(\mathrm{~s}, 1 \mathrm{H}), 8.13-$ $8.07(\mathrm{~m}, 1 \mathrm{H}), 7.58-7.47(\mathrm{~m}, 2 \mathrm{H}), 7.16-7.06(\mathrm{~m}, 2 \mathrm{H}), 7.06-7.00(\mathrm{~m}, 2 \mathrm{H}), 6.98-6.90(\mathrm{~m}, 1 \mathrm{H}), 3.87(\mathrm{~s}$, 3H), $3.77-3.69$ (m, 4H), $3.17-3.09$ (m, 4H). ${ }^{13} \mathrm{C}$ NMR (101 MHz, DMSO) $\delta 165.59,162.67,161.45$, 150.56, 150.47, 144.15, 130.76, 127.15, 124.77, 123.46, 121.44, 120.14, 116.58, 111.25, 90.90, 55.94, 49.85, 47.28. HRMS (ESI+) $\mathrm{m} / \mathrm{z}$ found $[\mathrm{M}+\mathrm{H}]^{+} 470.1491, \mathrm{C}_{22} \mathrm{H}_{24} \mathrm{~N}_{5} \mathrm{O}_{5} \mathrm{~S}$ requires 470.1493.

58: ${ }^{1} \mathrm{H}$ NMR (400 MHz, DMSO- $\left.d_{6}\right) \delta 12.65$ (br s, $\left.1 \mathrm{H}\right), 12.50(\mathrm{~s}, 1 \mathrm{H}), 12.13(\mathrm{~s}, 1 \mathrm{H}), 8.40(\mathrm{~d}, \mathrm{~J}=2.7 \mathrm{~Hz}$, $1 \mathrm{H}), 8.20(\mathrm{~s}, 1 \mathrm{H}), 8.15-8.09(\mathrm{~m}, 1 \mathrm{H}), 7.90(\mathrm{dd}, \mathrm{J}=9.2,2.8 \mathrm{~Hz}, 1 \mathrm{H}), 7.16-7.02(\mathrm{~m}, 3 \mathrm{H}), 6.99-6.91(\mathrm{~m}$, $1 \mathrm{H}), 4.08-4.00(\mathrm{~m}, 4 \mathrm{H}), 3.86(\mathrm{~s}, 3 \mathrm{H}), 3.14-3.06(\mathrm{~m}, 4 \mathrm{H}) .{ }^{13} \mathrm{C}$ NMR $(101 \mathrm{MHz}, \mathrm{DMSO}) \delta 166.03$, $162.66,153.29,150.74,150.42$, 140.04, 131.47, 127.12, 126.54, 124.82, 123.37, 120.16, 111.25, 107.72, 90.72, 55.94, 50.13, 43.78. HRMS (ESI+) $\mathrm{m} / \mathrm{z}$ found $[\mathrm{M}+\mathrm{H}]^{+} 471.1446, \mathrm{C}_{21} \mathrm{H}_{23} \mathrm{~N}_{6} \mathrm{O}_{5} \mathrm{~S}$ requires 471.1445.

59: ${ }^{1} \mathrm{H}$ NMR (400 MHz, DMSO- $d_{6}$ ) $\delta 12.68$ (br s, $\left.1 \mathrm{H}\right), 12.43$ (s, 1H), 12.04 (s, 1H), 8.69 (s, 2H), 8.21 (s, $1 \mathrm{H}), 8.13(\mathrm{dd}, \mathrm{J}=8.1,1.5 \mathrm{~Hz}, 1 \mathrm{H}), 7.17-7.06(\mathrm{~m}, 2 \mathrm{H}), 7.00-6.91(\mathrm{~m}, 1 \mathrm{H}), 4.27-4.14(\mathrm{~m}, 4 \mathrm{H}), 3.86(\mathrm{~s}$, $3 \mathrm{H}), 3.19-3.12(\mathrm{~m}, 4 \mathrm{H}) .{ }^{13} \mathrm{C}$ NMR (101 MHz, DMSO) $\delta 166.36,162.62,161.45,157.13,151.36$, 150.96, 150.36, 127.07, 124.88, 124.46, 123.30, 120.17, 111.25, 90.49, 55.93, 50.45, 42.54. HRMS (ESI+) $\mathrm{m} / \mathrm{z}$ found $[\mathrm{M}+\mathrm{H}]^{+} 472.1398, \mathrm{C}_{20} \mathrm{H}_{22} \mathrm{~N}_{7} \mathrm{O}_{5} \mathrm{~S}$ requires 472.1398 .

60: ${ }^{1} \mathrm{H}$ NMR (400 MHz, DMSO- $\left.d_{6}\right) \delta 12.92(\mathrm{~s}, 1 \mathrm{H}), 12.14(\mathrm{~s}, 1 \mathrm{H}), 11.37$ (br s, $\left.1 \mathrm{H}\right), 7.56-7.46(\mathrm{~m}, 2 \mathrm{H})$, $7.35-7.24(\mathrm{~m}, 3 \mathrm{H}), 7.16(\mathrm{dd}, \mathrm{J}=8.5,1.4 \mathrm{~Hz}, 1 \mathrm{H}), 7.06-6.97(\mathrm{~m}, 3 \mathrm{H}), 5.95(\mathrm{~d}, \mathrm{~J}=7.5 \mathrm{~Hz}, 1 \mathrm{H}), 3.82(\mathrm{~s}$, $3 \mathrm{H}), 3.75-3.68(\mathrm{~m}, 4 \mathrm{H}), 3.17-3.10(\mathrm{~m}, 4 \mathrm{H}) .{ }^{13} \mathrm{C}$ NMR (101 MHz, DMSO) $\delta 167.24,163.80,158.55$, 153.14, 143.97, 137.02, 131.12, 127.17, 126.42, 125.67, 121.32, 120.61, 116.63, 112.28, 96.15, 94.93, 55.68, 49.88, 47.36. HRMS (ESI+) $\mathrm{m} / \mathrm{z}$ found $[\mathrm{M}+\mathrm{H}]^{+} 469.1541, \mathrm{C}_{23} \mathrm{H}_{25} \mathrm{~N}_{4} \mathrm{O}_{5} \mathrm{~S}$ requires 469.1540 .

61: ${ }^{1} \mathrm{H}$ NMR (400 MHz, DMSO- $\left.d_{6}\right) \delta 12.85(\mathrm{~s}, 1 \mathrm{H}), 11.95(\mathrm{~s}, 1 \mathrm{H}), 11.43(\mathrm{~s}, 1 \mathrm{H}), 8.69(\mathrm{~s}, 2 \mathrm{H}), 7.35(\mathrm{~d}, \mathrm{~J}=$ $7.5 \mathrm{~Hz}, 1 \mathrm{H}), 7.32-7.25(\mathrm{~m}, 2 \mathrm{H}), 7.16(\mathrm{dd}, \mathrm{J}=8.4,1.2 \mathrm{~Hz}, 1 \mathrm{H}), 7.04-6.98(\mathrm{~m}, 1 \mathrm{H}), 5.94(\mathrm{~d}, \mathrm{~J}=7.4 \mathrm{~Hz}$, $1 \mathrm{H}), 4.26-4.15(\mathrm{~m}, 4 \mathrm{H}), 3.81(\mathrm{~s}, 3 \mathrm{H}), 3.18-3.11(\mathrm{~m}, 4 \mathrm{H}) .{ }^{13} \mathrm{C}$ NMR (101 MHz, DMSO) $\delta 167.94$, 163.81, 158.66, 156.98, 153.16, 151.04, 137.46, 127.36, 126.22, 125.74, 124.77, 120.63, 112.31, 96.11, 94.43, 55.68, 50.43, 42.55. HRMS (ESI+) $\mathrm{m} / \mathrm{z}$ found $[\mathrm{M}+\mathrm{H}]^{+} 471.1445, \mathrm{C}_{21} \mathrm{H}_{23} \mathrm{~N}_{6} \mathrm{O}_{5} \mathrm{~S}$ requires 471.1445 .

62: $\mathrm{HRMS}(\mathrm{ESI}+) \mathrm{m} / \mathrm{z}$ found $[\mathrm{M}+\mathrm{H}]^{+}$307.1443, $\mathrm{C}_{19} \mathrm{H}_{19} \mathrm{~N}_{2} \mathrm{O}_{2}$ requires 307.1441.

63: ${ }^{1} \mathrm{H}$ NMR (400 MHz, DMSO- $\left.d_{6}\right) \delta 10.45(\mathrm{~s}, 1 \mathrm{H}), 7.94(\mathrm{~d}, \mathrm{~J}=8.8 \mathrm{~Hz}, 1 \mathrm{H}), 7.88(\mathrm{~s}, 1 \mathrm{H}), 7.80(\mathrm{~d}, \mathrm{~J}=7.7$ $\mathrm{Hz}, 1 \mathrm{H}), 7.74(\mathrm{~d}, \mathrm{~J}=3.0 \mathrm{~Hz}, 1 \mathrm{H}), 7.70(\mathrm{~d}, \mathrm{~J}=8.5 \mathrm{~Hz}, 1 \mathrm{H}), 7.49(\mathrm{t}, \mathrm{J}=7.8 \mathrm{~Hz}, 1 \mathrm{H}), 7.35$ (t, J = $7.5 \mathrm{~Hz}$, $1 \mathrm{H}), 7.05(\mathrm{dd}, \mathrm{J}=8.9,3.1 \mathrm{~Hz}, 1 \mathrm{H}), 3.29-3.23(\mathrm{~m}, 4 \mathrm{H}), 2.02-1.92(\mathrm{~m}, 4 \mathrm{H}) .{ }^{13} \mathrm{C}$ NMR $(101 \mathrm{MHz}$, DMSO) $\delta 156.14,154.44,148.55,141.48,140.44,130.98,127.18,126.99,123.75,122.87,120.19$, 115.72, 111.92, 110.12, 47.28, 24.85. HRMS (ESI+) $\mathrm{m} / \mathrm{z}$ found $[\mathrm{M}+\mathrm{H}]^{+} 308.1392, \mathrm{C}_{18} \mathrm{H}_{18} \mathrm{~N}_{3} \mathrm{O}_{2}$ requires 308.1399 . 
64: ${ }^{1} \mathrm{H}$ NMR (400 MHz, DMSO- $\left.d_{6}\right) \delta 10.36(\mathrm{~s}, 1 \mathrm{H}), 8.42(\mathrm{~d}, \mathrm{~J}=2.6 \mathrm{~Hz}, 1 \mathrm{H}), 7.87(\mathrm{dd}, \mathrm{J}=9.0,2.6 \mathrm{~Hz}$, $1 \mathrm{H}), 7.83-7.79(\mathrm{~m}, 1 \mathrm{H}), 7.73-7.66(\mathrm{~m}, 2 \mathrm{H}), 7.53-7.46(\mathrm{~m}, 1 \mathrm{H}), 7.36(\mathrm{t}, \mathrm{J}=7.5 \mathrm{~Hz}, 1 \mathrm{H}), 6.47(\mathrm{~d}, \mathrm{~J}=$ $9.0 \mathrm{~Hz}, 1 \mathrm{H}), 3.41-3.34(\mathrm{~m}, 4 \mathrm{H}), 1.99-1.90(\mathrm{~m}, 4 \mathrm{H}) .{ }^{13} \mathrm{C}$ NMR (101 MHz, DMSO) $\delta 156.35,154.38$, 154.34, 149.02, 141.18, 131.19, 127.21, 126.98, 123.92, 123.80, 122.82, 111.86, 110.13, 105.62, 46.50, 25.03. HRMS (ESI+) $\mathrm{m} / \mathrm{z}$ found $[\mathrm{M}+\mathrm{H}]^{+} 308.1390, \mathrm{C}_{18} \mathrm{H}_{18} \mathrm{~N}_{3} \mathrm{O}_{2}$ requires 308.1399.

65: $7.48(\mathrm{~s}, 1 \mathrm{H}), 7.27(\mathrm{dd}, \mathrm{J}=9.4,2.7 \mathrm{~Hz}, 1 \mathrm{H}), 7.24(\mathrm{~d}, \mathrm{~J}=2.1 \mathrm{~Hz}, 1 \mathrm{H}), 7.21-7.14(\mathrm{~m}, 3 \mathrm{H}), 7.11(\mathrm{~d}, \mathrm{~J}=$ $2.3 \mathrm{~Hz}, 1 \mathrm{H}), 6.89-6.84(\mathrm{~m}, 2 \mathrm{H}), 6.51(\mathrm{~s}, 1 \mathrm{H}), 5.1-4.80(\mathrm{~m}, 1 \mathrm{H}), 4.34-4.24(\mathrm{~m}, 2 \mathrm{H}), 4.20-4.03(\mathrm{~m}$, $3 \mathrm{H}), 3.90-3.82(\mathrm{~m}, 1 \mathrm{H}), 3.57(\mathrm{~s}, 3 \mathrm{H}), 3.03(\mathrm{dd}, \mathrm{J}=6.3,3.7 \mathrm{~Hz}, 4 \mathrm{H}), 2.66-2.52(\mathrm{~m}, 2 \mathrm{H}), 2.49-2.38(\mathrm{~m}$, $6 \mathrm{H}), 2.20(\mathrm{~s}, 3 \mathrm{H}), 1.85-1.64(\mathrm{~m}, 4 \mathrm{H}) .{ }^{13} \mathrm{C}$ NMR (101 MHz, DMSO) $\delta 162.31,159.88,158.91,156.71$, 144.75 (d), 141.97 (d), 133.50, 132.95, 132.89 (d), 131.68, 125.50, 122.08, 121.07, 120.97, 118.51, 117.81 (d), 116.89, 116.41, 115.55 (d), 114.18 (d), 111.44, 109.92, 63.88, 56.87, 54.69, 49.54, 48.74, 45.76, 37.20, 23.12, 22.54, 22.39, 20.88. HRMS (ESI+) $\mathrm{m} / \mathrm{z}$ found $[\mathrm{M}+\mathrm{H}]^{+} 611.3135, \mathrm{C}_{35} \mathrm{H}_{40} \mathrm{FN}_{6} \mathrm{O}_{3}=$ 611.3140 .

66: ${ }^{1} \mathrm{H}$ NMR (400 MHz, DMSO- $\left.d_{6}\right) \delta 8.54(\mathrm{~d}, \mathrm{~J}=2.1 \mathrm{~Hz}, 1 \mathrm{H}), 8.35(\mathrm{~s}, 1 \mathrm{H}), 7.85(\mathrm{~d}, \mathrm{~J}=3.0 \mathrm{~Hz}, 1 \mathrm{H}), 7.35$ $(\mathrm{dd}, \mathrm{J}=7.8,2.7 \mathrm{~Hz}, 2 \mathrm{H}), 7.31(\mathrm{dd}, \mathrm{J}=9.4,2.6 \mathrm{~Hz}, 1 \mathrm{H}), 7.21(\mathrm{~d}, \mathrm{~J}=9.0 \mathrm{~Hz}, 1 \mathrm{H}), 7.17(\mathrm{dd}, \mathrm{J}=9.3,2.7 \mathrm{~Hz}$, 1H), $6.52(\mathrm{~s}, 1 \mathrm{H}), 4.84(\mathrm{t}, \mathrm{J}=4.6 \mathrm{~Hz}, 1 \mathrm{H}), 4.31(\mathrm{~d}, \mathrm{~J}=4.5 \mathrm{~Hz}, 2 \mathrm{H}), 4.22-4.06(\mathrm{~m}, 3 \mathrm{H}), 3.94-3.83(\mathrm{~m}$, $1 \mathrm{H}), 3.58(\mathrm{~s}, 3 \mathrm{H}), 3.08-2.99(\mathrm{~m}, 4 \mathrm{H}), 2.59(\mathrm{q}, \mathrm{J}=7.5,6.9 \mathrm{~Hz}, 2 \mathrm{H}), 2.44(\mathrm{~m}, 6 \mathrm{H}), 2.20(\mathrm{~s}, 3 \mathrm{H}), 1.85-$ $1.63(\mathrm{~m}, 4 \mathrm{H}) .{ }^{13} \mathrm{C}$ NMR (101 MHz, DMSO) $\delta 162.34,159.91,158.90,156.66,148.51,144.78$ (d), 142.04 (d), 141.00, 133.91, 133.23 (d), 131.73, 130.70, 126.98, 126.86, 122.13, 118.55, 117.69, 116.25, 115.72 (d), 114.33 (d), 112.89, 111.47, 56.76, 54.53, 49.59, 48.82, 45.79, 37.45, 23.15, 22.57, 22.41, 20.90. HRMS (ESI+) $\mathrm{m} / \mathrm{z}$ found $[\mathrm{M}+\mathrm{H}]^{+} 612.3089, \mathrm{C}_{34} \mathrm{H}_{39} \mathrm{FN}_{7} \mathrm{O}_{3}$ requires 612.3098.

67: H NMR (400 MHz, DMSO- $\left.d_{6}\right) \delta 8.65(\mathrm{~d}, \mathrm{~J}=2.2 \mathrm{~Hz}, 1 \mathrm{H}), 8.58(\mathrm{~s}, 1 \mathrm{H}), 7.54(\mathrm{~d}, \mathrm{~J}=9.9 \mathrm{~Hz}, 1 \mathrm{H}), 7.40$ $(\mathrm{d}, \mathrm{J}=2.5 \mathrm{~Hz}, 1 \mathrm{H}), 7.36-7.27(\mathrm{~m}, 2 \mathrm{H}), 7.17(\mathrm{dd}, \mathrm{J}=9.3,2.8 \mathrm{~Hz}, 1 \mathrm{H}), 6.52(\mathrm{~s}, 1 \mathrm{H}), 4.80(\mathrm{t}, \mathrm{J}=4.7 \mathrm{~Hz}$, $1 \mathrm{H}), 4.36-4.26(\mathrm{~m}, 2 \mathrm{H}), 4.21-4.07(\mathrm{~m}, 3 \mathrm{H}), 3.94-3.83(\mathrm{~m}, 1 \mathrm{H}), 3.59(\mathrm{~s}, 3 \mathrm{H}), 3.41(\mathrm{t}, \mathrm{J}=4.9 \mathrm{~Hz}, 4 \mathrm{H})$, $2.68-2.52(\mathrm{~m}, 2 \mathrm{H}), 2.46(\mathrm{t}, \mathrm{J}=6.0 \mathrm{~Hz}, 2 \mathrm{H}), 2.39(\mathrm{t}, \mathrm{J}=5.0 \mathrm{~Hz}, 4 \mathrm{H}), 2.20(\mathrm{~s}, 3 \mathrm{H}), 1.85-1.62(\mathrm{~m}, 4 \mathrm{H})$. ${ }^{13} \mathrm{C}$ NMR (101 MHz, DMSO) $\delta$ 162.30, 159.86, 158.86, 156.63, 156.18, 151.82, 144.75, 144.68 (d), 141.81 (d), 133.30 (d), 131.67, 130.11, 127.60, 122.11, 120.92, 118.50, 118.36, 117.51, 117.17, 115.70 (d), 114.23 (d), 111.43, 56.75, 54.28, 49.53, 45.82, 45.45, 37.43, 23.12, 22.55, 22.39, 20.87. HRMS (ESI+) $\mathrm{m} / \mathrm{z}$ found $[\mathrm{M}+\mathrm{H}]^{+}$613.3044, $\mathrm{C}_{33} \mathrm{H}_{38} \mathrm{FN}_{8} \mathrm{O}_{3}$ requires 613.3045 .

68: ${ }^{1} \mathrm{H}$ NMR (400 MHz, DMSO- $\left.d_{6}\right) \delta 8.46(\mathrm{~s}, 1 \mathrm{H}), 7.49(\mathrm{~s}, 1 \mathrm{H}), 7.37-7.28(\mathrm{~m}, 2 \mathrm{H}), 7.25(\mathrm{~d}, \mathrm{~J}=2.3 \mathrm{~Hz}$, $1 \mathrm{H}), 7.21-7.16(\mathrm{~m}, 2 \mathrm{H}), 7.05(\mathrm{~d}, \mathrm{~J}=2.2 \mathrm{~Hz}, 1 \mathrm{H}), 6.90-6.84(\mathrm{~m}, 2 \mathrm{H}), 4.64(\mathrm{t}, \mathrm{J}=4.8 \mathrm{~Hz}, 1 \mathrm{H}), 4.24(\mathrm{~d}$, $\mathrm{J}=4.3 \mathrm{~Hz}, 2 \mathrm{H}), 3.57(\mathrm{~s}, 3 \mathrm{H}), 3.03(\mathrm{dd}, \mathrm{J}=6.1,3.7 \mathrm{~Hz}, 4 \mathrm{H}), 2.97-2.90(\mathrm{~m}, 2 \mathrm{H}), 2.87-2.80(\mathrm{~m}, 2 \mathrm{H})$, $2.45-2.39(\mathrm{~m}, 4 \mathrm{H}), 2.20(\mathrm{~s}, 3 \mathrm{H}), 1.94-1.80(\mathrm{~m}, 4 \mathrm{H}) .{ }^{13} \mathrm{C}$ NMR (101 MHz, DMSO) $\delta 161.75,159.32$, 156.71, 156.46, 146.61, 146.61, 143.17 (d), 141.96 (d), 133.58 (d), 132.50, 131.65, 125.59, 121.14, 117.38, 117.03 (d), 116.41, 114.89 (d), 109.82, 56.39, 54.68, 48.73, 45.75, 37.20, 25.18, 23.09, 22.55, 21.29. HRMS (ESI+) $\mathrm{m} / \mathrm{z}$ found $[\mathrm{M}+\mathrm{H}]^{+}$627.2544, $\mathrm{C}_{34} \mathrm{H}_{36} \mathrm{FN}_{6} \mathrm{O}_{3} \mathrm{~S}$ requires 627.2548.

69: ${ }^{1} \mathrm{H}$ NMR (400 MHz, DMSO- $\left.d_{6}\right) \delta 8.54(\mathrm{~d}, \mathrm{~J}=2.4 \mathrm{~Hz}, 1 \mathrm{H}), 8.47(\mathrm{~s}, 1 \mathrm{H}), 8.37(\mathrm{~s}, 1 \mathrm{H}), 7.86(\mathrm{~d}, \mathrm{~J}=3.0$ $\mathrm{Hz}, 1 \mathrm{H}), 7.41-7.29(\mathrm{~m}, 4 \mathrm{H}), 7.21(\mathrm{~d}, \mathrm{~J}=9.0 \mathrm{~Hz}, 1 \mathrm{H}), 4.57(\mathrm{t}, \mathrm{J}=4.9 \mathrm{~Hz}, 1 \mathrm{H}), 4.29(\mathrm{~d}, \mathrm{~J}=4.9 \mathrm{~Hz}, 2 \mathrm{H})$, $3.58(\mathrm{~s}, 3 \mathrm{H}), 3.03(\mathrm{t}, \mathrm{J}=4.9 \mathrm{~Hz}, 4 \mathrm{H}), 2.97-2.90(\mathrm{~m}, 2 \mathrm{H}), 2.88-2.81(\mathrm{~m}, 2 \mathrm{H}), 2.43(\mathrm{t}, \mathrm{J}=5.0 \mathrm{~Hz}, 4 \mathrm{H})$, $2.20(\mathrm{~s}, 3 \mathrm{H}), 1.94-1.80(\mathrm{~m}, 4 \mathrm{H}) .{ }^{13} \mathrm{C}$ NMR $(101 \mathrm{MHz}, \mathrm{DMSO}) \delta 161.73,159.28,156.61,156.45$, $148.49,146.57,143.12$ (d), 141.93 (d), 140.97, 138.58, 133.85, 133.57, 132.78 (d), 132.75, 132.45, 131.67, 130.68, 126.92 (d), 117.24 (d), 117.01, 116.12, 115.11, 114.88, 112.91, 56.42, 54.51, 48.79, 45.76, 37.39, 25.19, 23.10, 22.56, 21.30. HRMS (ESI+) $\mathrm{m} / \mathrm{z}$ found $[\mathrm{M}+\mathrm{H}]^{+} 628.2506, \mathrm{C}_{33} \mathrm{H}_{35} \mathrm{FN}_{7} \mathrm{O}_{3} \mathrm{~S}$ requires 628.2501 . 
70: ${ }^{13} \mathrm{C}$ NMR (101 MHz, DMSO) $\delta 8.64(\mathrm{~d}, \mathrm{~J}=2.2 \mathrm{~Hz}, 1 \mathrm{H}), 8.59(\mathrm{~s}, 1 \mathrm{H}), 8.47(\mathrm{~s}, 1 \mathrm{H}), 7.55(\mathrm{~d}, \mathrm{~J}=9.6$ $\mathrm{Hz}, 1 \mathrm{H}), 7.43-7.37(\mathrm{~m}, 2 \mathrm{H}), 7.32(\mathrm{~d}, \mathrm{~J}=2.9 \mathrm{~Hz}, 1 \mathrm{H}), 7.30(\mathrm{~d}, \mathrm{~J}=3.4 \mathrm{~Hz}, 1 \mathrm{H}), 4.57(\mathrm{t}, \mathrm{J}=5.2 \mathrm{~Hz}, 1 \mathrm{H})$, $4.29(\mathrm{~d}, \mathrm{~J}=4.9 \mathrm{~Hz}, 2 \mathrm{H}), 3.60(\mathrm{~s}, 3 \mathrm{H}), 3.42(\mathrm{t}, \mathrm{J}=5.0 \mathrm{~Hz}, 4 \mathrm{H}), 2.98-2.90(\mathrm{~m}, 2 \mathrm{H}), 2.88-280(\mathrm{~m}, 2 \mathrm{H})$, $2.40(\mathrm{t}, \mathrm{J}=5.1 \mathrm{~Hz}, 4 \mathrm{H}), 2.21(\mathrm{~s}, 3 \mathrm{H}), 1.94-1.80(\mathrm{~m}, 4 \mathrm{H}) .{ }^{13} \mathrm{C}$ NMR $(101 \mathrm{MHz}, \mathrm{DMSO}) \delta 161.69$, 159.25, 156.61, 156.41, 156.18, 151.82, 146.55, 143.07 (d), 141.69 (d), 133.57, 132.84 (d), 132.44, 131.66, 130.16, 127.72, 120.94, 118.13, 117.14, 116.98, 115.15, 114.92, 56.50, 54.28, 45.82, 45.45, 37.42, 25.18, 23.10, 22.56, 21.30. HRMS (ESI+) $\mathrm{m} / \mathrm{z}$ found $[\mathrm{M}+\mathrm{H}]^{+} 629.2458, \mathrm{C}_{32} \mathrm{H}_{34} \mathrm{FN}_{8} \mathrm{O}_{3} \mathrm{~S}$ requires 629.2453 .

71: ${ }^{1} \mathrm{H}$ NMR $\left(400 \mathrm{MHz}, \mathrm{DMSO}-d_{6}\right) \delta 8.55(\mathrm{~d}, \mathrm{~J}=2.4 \mathrm{~Hz}, 1 \mathrm{H}), 8.36(\mathrm{~s}, 1 \mathrm{H}), 7.86(\mathrm{~d}, \mathrm{~J}=3.0 \mathrm{~Hz}, 1 \mathrm{H}), 7.39$ $-7.33(\mathrm{~m}, 2 \mathrm{H}), 7.31(\mathrm{dd}, \mathrm{J}=9.4,2.7 \mathrm{~Hz}, 1 \mathrm{H}), 7.21(\mathrm{~d}, \mathrm{~J}=9.0 \mathrm{~Hz}, 1 \mathrm{H}), 7.17(\mathrm{dd}, \mathrm{J}=9.4,2.8 \mathrm{~Hz}, 1 \mathrm{H})$, $6.52(\mathrm{~s}, 1 \mathrm{H}), 4.85(\mathrm{t}, \mathrm{J}=4.5 \mathrm{~Hz}, 1 \mathrm{H}), 4.55(\mathrm{t}, \mathrm{J}=6.5 \mathrm{~Hz}, 2 \mathrm{H}), 4.45(\mathrm{t}, \mathrm{J}=6.0 \mathrm{~Hz}, 2 \mathrm{H}), 4.31(\mathrm{~d}, \mathrm{~J}=4.5 \mathrm{~Hz}$, $2 \mathrm{H}), 4.21-4.06(\mathrm{~m}, 3 \mathrm{H}), 3.93-3.83(\mathrm{~m}, 1 \mathrm{H}), 3.58(\mathrm{~s}, 3 \mathrm{H}), 3.42(\mathrm{dd}, \mathrm{J}=12.4,6.1 \mathrm{~Hz}, 1 \mathrm{H}), 3.06(\mathrm{t}, \mathrm{J}=$ $5.0 \mathrm{~Hz}, 4 \mathrm{H}), 2.67-2.53(\mathrm{~m}, 2 \mathrm{H}), 2.46(\mathrm{t}, \mathrm{J}=6.0 \mathrm{~Hz}, 2 \mathrm{H}), 2.38(\mathrm{t}, \mathrm{J}=5.0 \mathrm{~Hz}, 4 \mathrm{H}), 1.84-1.63(\mathrm{~m}, 4 \mathrm{H})$. ${ }^{13} \mathrm{C}$ NMR (101 MHz, DMSO) $\delta 162.32,159.88,158.86,156.63,148.60,144.76$ (d), 142.03 (d), 141.97, $140.91,133.95,133.25,131.68,130.67,127.04,126.86,122.12,118.52,117.64,116.28,115.70$ (d), 114.31 (d), 112.89, 111.43, 74.35, 58.47, 56.73, 49.57, 48.92, 48.67, 37.42, 23.12, 22.55, 22.39, 20.88. HRMS (ESI+) $\mathrm{m} / \mathrm{z}$ found $[\mathrm{M}+\mathrm{H}]^{+} 654.3194, \mathrm{C}_{36} \mathrm{H}_{41} \mathrm{FN}_{7} \mathrm{O}_{4}$ requires 654.3199.

72: ${ }^{1} \mathrm{H}$ NMR (400 MHz, DMSO- $\left.d_{6}\right) \delta 8.47(\mathrm{~d}, \mathrm{~J}=2.3 \mathrm{~Hz}, 1 \mathrm{H}), 8.35(\mathrm{~s}, 2 \mathrm{H}), 8.07(\mathrm{~s}, 1 \mathrm{H}), 7.45$ (d, J = 2.2 $\mathrm{Hz}, 1 \mathrm{H}), 7.32(\mathrm{dd}, \mathrm{J}=9.4,2.8 \mathrm{~Hz}, 1 \mathrm{H}), 7.19(\mathrm{dd}, \mathrm{J}=9.4,2.7 \mathrm{~Hz}, 1 \mathrm{H}), 6.52(\mathrm{~s}, 1 \mathrm{H}), 4.86(\mathrm{t}, \mathrm{J}=4.5 \mathrm{~Hz}$, $1 \mathrm{H}), 4.55(\mathrm{t}, \mathrm{J}=6.5 \mathrm{~Hz}, 2 \mathrm{H}), 4.45(\mathrm{t}, \mathrm{J}=6.1 \mathrm{~Hz}, 2 \mathrm{H}), 4.30(\mathrm{~d}, \mathrm{~J}=4.5 \mathrm{~Hz}, 2 \mathrm{H}), 4.22-4.06(\mathrm{~m}, 3 \mathrm{H}), 3.94$ $-3.83(\mathrm{~m}, 1 \mathrm{H}), 3.60(\mathrm{~s}, 3 \mathrm{H}), 3.45(\mathrm{q}, \mathrm{J}=6.3 \mathrm{~Hz}, 1 \mathrm{H}), 3.13(\mathrm{dd}, \mathrm{J}=6.4,3.7 \mathrm{~Hz}, 4 \mathrm{H}), 2.68-2.53(\mathrm{~m}, 2 \mathrm{H})$, $2.46(\mathrm{t}, \mathrm{J}=6.0 \mathrm{~Hz}, 2 \mathrm{H}), 2.40(\mathrm{t}, \mathrm{J}=5.0 \mathrm{~Hz}, 4 \mathrm{H}), 1.85-1.63(\mathrm{~m}, 4 \mathrm{H}) .{ }^{13} \mathrm{C}$ NMR (101 MHz, DMSO) $\delta$ $162.30,159.86,158.84,156.38,152.91,146.17,144.74$ (d), 141.61 (d), 139.02, 133.29 (d), 131.69, 129.06, 128.21, 122.09, 118.51, 117.76, 117.56, 115.75 (d), 114.47 (d), 111.41, 74.32, 58.42, 56.66, $49.55,48.66,48.07,37.49,23.12,22.54,22.39,20.88$. HRMS (ESI+) $\mathrm{m} / \mathrm{z}$ found $[\mathrm{M}+\mathrm{H}]^{+} 655.3153$, $\mathrm{C}_{35} \mathrm{H}_{40} \mathrm{FN}_{8} \mathrm{O}_{4}$ requires 655.3151 .

73: ${ }^{1} \mathrm{H}$ NMR (400 MHz, DMSO-d $) \delta 8.65(\mathrm{~d}, \mathrm{~J}=2.3 \mathrm{~Hz}, 1 \mathrm{H}), 8.58(\mathrm{~s}, 1 \mathrm{H}), 7.55(\mathrm{~d}, \mathrm{~J}=9.8 \mathrm{~Hz}, 1 \mathrm{H}), 7.40$ $(\mathrm{d}, \mathrm{J}=2.4 \mathrm{~Hz}, 1 \mathrm{H}), 7.32$ (dd, J = 9.6, 2.6 Hz, 2H), 7.17 (dd, J = 9.3, $2.8 \mathrm{~Hz}, 1 \mathrm{H}), 6.52$ (s, 1H), 4.79 (dd, J $=5.6,3.8 \mathrm{~Hz}, 1 \mathrm{H}), 4.55(\mathrm{t}, \mathrm{J}=6.5 \mathrm{~Hz}, 2 \mathrm{H}), 4.47(\mathrm{t}, \mathrm{J}=6.0 \mathrm{~Hz}, 2 \mathrm{H}), 4.38-4.23(\mathrm{~m}, 2 \mathrm{H}), 4.22-4.05(\mathrm{~m}$, $3 \mathrm{H}), 3.94-3.81(\mathrm{~m}, 1 \mathrm{H}), 3.59(\mathrm{~s}, 3 \mathrm{H}), 3.48-3.36(\mathrm{~m}, 5 \mathrm{H}), 2.67-2.51(\mathrm{~m}, 2 \mathrm{H}), 2.46(\mathrm{t}, \mathrm{J}=6.0 \mathrm{~Hz}, 2 \mathrm{H})$, $2.35(\mathrm{t}, \mathrm{J}=5.1 \mathrm{~Hz}, 4 \mathrm{H}), 1.85-1.62(\mathrm{~m}, 4 \mathrm{H}) .{ }^{13} \mathrm{C}$ NMR (101 MHz, DMSO) $\delta 162.30,159.86,158.86$, 156.62, 156.10, 152.15, 151.86, 144.69 (d), 141.80 (d), 133.30 (d), 131.67, 130.10, 127.62, 122.11, 120.96, 118.49, 118.39, 117.50, 117.17, 115.71 (d), 114.22 (d), 111.42, 74.35, 58.48, 56.75, 49.53, 48.73, 45.26, 37.43, 23.12, 22.55, 22.39, 20.87. HRMS (ESI+) $\mathrm{m} / \mathrm{z}$ found $[\mathrm{M}+\mathrm{H}]^{+} 655.3149, \mathrm{C}_{35} \mathrm{H}_{40} \mathrm{FN}_{8} \mathrm{O}_{4}=$ 655.3151 .

74: ${ }^{1} \mathrm{H}$ NMR (400 MHz, DMSO- $\left.d_{6}\right) \delta 10.99(\mathrm{~d}, \mathrm{~J}=4.8 \mathrm{~Hz}, 1 \mathrm{H}), 9.41(\mathrm{~s}, 1 \mathrm{H}), 8.15(\mathrm{~s}, 1 \mathrm{H}), 8.09(\mathrm{~d}, \mathrm{~J}=5.1$ $\mathrm{Hz}, 1 \mathrm{H}), 7.92-7.87(\mathrm{~m}, 2 \mathrm{H}), 7.67-7.55(\mathrm{~m}, 3 \mathrm{H}), 7.39$ (dd, J = 9.1, 3.0 Hz, 1H), 7.16 (dd, J =5.6, 1.9 Hz, 1H), $3.15-3.07(\mathrm{~m}, 4 \mathrm{H}), 2.62(\mathrm{t}, \mathrm{J}=5.0 \mathrm{~Hz}, 4 \mathrm{H}), 2.33(\mathrm{~s}, 3 \mathrm{H})$. HRMS (ESI+) $\mathrm{m} / \mathrm{z}$ found $[\mathrm{M}+\mathrm{H}]^{+}$ 457.1309, $\mathrm{C}_{22} \mathrm{H}_{23} \mathrm{Cl}_{2} \mathrm{~N}_{6} \mathrm{O}$ requires 457.1305.

75: ${ }^{1} \mathrm{H}$ NMR $\left(400 \mathrm{MHz}, \mathrm{DMSO}-d_{6}\right) \delta 11.12(\mathrm{~s}, 1 \mathrm{H}), 9.61(\mathrm{~s}, 1 \mathrm{H}), 8.11(\mathrm{~d}, \mathrm{~J}=5.6 \mathrm{~Hz}, 1 \mathrm{H}), 8.04(\mathrm{~d}, \mathrm{~J}=1.9$ $\mathrm{Hz}, 1 \mathrm{H}), 7.67(\mathrm{~d}, \mathrm{~J}=9.8 \mathrm{~Hz}, 1 \mathrm{H}), 7.60(\mathrm{~d}, \mathrm{~J}=8.0 \mathrm{~Hz}, 1 \mathrm{H}), 7.58(\mathrm{~d}, \mathrm{~J}=1.2 \mathrm{~Hz}, 1 \mathrm{H}), 7.51(\mathrm{dd}, \mathrm{J}=9.2,6.8$ $\mathrm{Hz}, 1 \mathrm{H}), 7.38-7.29(\mathrm{~m}, 2 \mathrm{H}), 3.47-3.40(\mathrm{~m}, 4 \mathrm{H}), 2.42(\mathrm{t}, \mathrm{J}=5.0 \mathrm{~Hz}, 4 \mathrm{H}), 2.22(\mathrm{~s}, 3 \mathrm{H}) .{ }^{13} \mathrm{C}$ NMR $(101$ MHz, DMSO) $\delta 163.32,157.22,155.74,151.63,148.93,146.96,136.47,132.01,131.48,128.69,120.95$, 
117.10, 107.08, 100.62, 54.74, 46.32, 45.97. HRMS (ESI+) m/z found $[\mathrm{M}+\mathrm{H}]^{+} 458.1261, \mathrm{C}_{21} \mathrm{H}_{22} \mathrm{Cl}_{2} \mathrm{~N}_{7} \mathrm{O}$ requires 458.1257 . 


\section{In Silico Modeling and Associated Data}

Bond dissociation enthalpies (BDE's) of aniline N-H and phenolic O-H bonds were calculated using formula (1):

$$
\mathrm{BDE}_{\mathrm{R}-\mathrm{H}}=\mathrm{H}_{\mathrm{R}}+\mathrm{H}_{\mathrm{H}}-\mathrm{H}_{\mathrm{R}-\mathrm{H}}
$$

where $\mathrm{H}_{\mathrm{R}}, \mathrm{H}_{\mathrm{H}}$ and $\mathrm{H}_{\mathrm{R}-\mathrm{H}}$ represent the total enthalpies of the radical $\mathrm{R} \cdot(\mathrm{Ar}-\mathrm{N} \cdot$ or Ar-O$\cdot$ ), hydrogen atom $\mathrm{H}$ or $(\mathrm{H} \cdot)$, and the parent molecule $\mathrm{R}-\mathrm{H}$. The total enthalpy of each species is calculated using formula (2):

$$
\mathrm{H}=\mathrm{E}_{0}+\mathrm{E}_{\text {thermal correction to enthalpy }}
$$

where $\mathrm{E}_{0}$ is the calculated total electronic energy at $0 \mathrm{~K}$ degree, $\mathrm{E}_{\text {thermal correction to enthalpy }}$ is the correction for translational, rotational and vibrational energies and RT at 298.15K.

The initial geometries of all fragments were generated by ligprep followed by minimization using Macromodel with OPLS3 force field as implemented in Maestro of the Schrodinger 2017-2 release. ${ }^{3}$ To identify lowest energy conformations as starting points, QM torsion scans ${ }^{4}$ were carried out for substituted aniline fragments along the $\mathrm{C}_{\mathrm{ar}}$-NR1R2 bond (number of steps $=36$, step size $=10^{0}$ ) using Gaussian software with MP2/6-311+G**//HF/6-31G* basis set. ${ }^{6}$ One or several representative low energy conformations (within $0.2 \mathrm{kcal} / \mathrm{mol}$ of the lowest energy conformation) were selected for each aniline fragment based on the torsional scan results, and corresponding aniline $\mathrm{N} \cdot$ and/or phenolic $\mathrm{O} \cdot$ radicals were generated from these conformations. For anilines with multiple N-H bonds, multiple radicals were generated and subjected to optimizations.

The selected low energy conformations of parent molecules, corresponding radicals and hydrogen atom were subjected to optimizations using Gaussian, ${ }^{5}$ with either restricted (for parent molecule) or unrestricted (for radicals) $\mathrm{HF} / 6-311+\mathrm{G}^{* *}$ in water solvent $(\mathrm{PCM})^{6}$ and DFT B3LYP/6-311+G** in gas phase followed by frequency calculations to confirm real minima were reached (no imaginary frequencies). ${ }^{7,8}$ A single point energy calculation was performed at the end using restricted (for parent molecule) or restricted open shell (for radicals) MP2/6-311+G** with PCM water solvent model for the HF optimized conformations, and restricted or restricted open shell DFT/B3-LYP 6-311+G** in gas phase 
for DFT optimized radical conformations. The energies from single point energy calculations and thermal corrections from frequency calculations were used to calculate BDEs for each aniline $\mathrm{N}-\mathrm{H}$ and phenolic O$\mathrm{H}$ bond. For fragments with multiple aniline N-H, the lowest BDE was chosen as the energy required for first step hydrogen abstraction. $\triangle \mathrm{BDEs}$ calculated with $\mathrm{MP} 2 / 6-311+\mathrm{G}^{* *}$ basis set provided slightly better correlation with observed relative intensity of GSH conjugates, and are shown in Table S4 to S6. 
Table S4: Total electronic energies, thermal corrections, and calculated $\triangle \mathrm{BDEs}$ for the parent and radical of 4-substituted anilines (entries 5 to $\mathbf{1 2}$ )

\begin{tabular}{|c|c|c|c|c|c|c|}
\hline \multirow[b]{2}{*}{ Entry } & \multicolumn{2}{|c|}{ Radical } & \multicolumn{2}{|c|}{ Parent } & \multirow[b]{2}{*}{$\begin{array}{c}\Delta \mathrm{BDE}^{\mathrm{a}} \\
(\mathrm{kcal} / \mathrm{mol})\end{array}$} & \multirow[b]{2}{*}{$\begin{array}{c}\text { GSH } \\
\text { conjugates }\end{array}$} \\
\hline & $\begin{array}{c}\text { Total } \\
\text { Electronic } \\
\text { Energy } \\
\text { (Hartree) }\end{array}$ & $\begin{array}{c}\text { Thermal } \\
\text { correction for } \\
\text { enthapy } \\
\text { (Hartree) }\end{array}$ & $\begin{array}{c}\text { Total } \\
\text { Electronic } \\
\text { Energy } \\
\text { (Hartree) }\end{array}$ & $\begin{array}{c}\text { Thermal } \\
\text { correction for } \\
\text { enthapy } \\
\text { (Hartree) }\end{array}$ & & \\
\hline 5 & -380.590804 & 0.165051 & -381.236655 & 0.181451 & 0 & +++ \\
\hline 6 & -396.621097 & 0.153189 & -397.270864 & 0.169092 & 2.77 & BLD \\
\hline 7 & -412.619574 & 0.139699 & -413.272819 & 0.156525 & 4.37 & BLD \\
\hline 8 & -717.046964 & 0.174635 & -717.695254 & 0.190544 & 1.84 & +++ \\
\hline 9 & -479.666937 & 0.157554 & -480.313962 & 0.173816 & 0.82 & BLD \\
\hline 10 & -839.650742 & 0.156348 & -840.298773 & 0.172742 & 1.37 & ++ \\
\hline 11 & -2952.497629 & 0.155872 & -2953.145647 & 0.172276 & 1.36 & ++ \\
\hline 12 & -472.631892 & 0.165239 & -473.278505 & 0.181984 & 0.26 & ++ \\
\hline
\end{tabular}

${ }^{\mathrm{a}} \triangle \mathrm{BDEs}$ of first hydrogen atom abstraction refers to the difference between the lowest BDE of any $\mathrm{N}-\mathrm{H}$ of a fragment vs. $\mathrm{N}-\mathrm{H}$ bond of $\mathrm{N}$-methylbenzene-1,4-diamine. 
Table S5. Total electronic energies, thermal corrections, and calculated $\triangle B D E s$ for the parent and radical of entries $\mathbf{1 3}$ to $\mathbf{2 0}$

\begin{tabular}{|c|c|c|c|c|c|c|}
\hline \multirow[b]{2}{*}{ Entry } & \multicolumn{2}{|c|}{ Radical } & \multicolumn{2}{|c|}{ Parent } & \multirow[b]{2}{*}{$\begin{array}{c}\Delta \mathrm{BDE}^{\mathrm{a}} \\
(\mathrm{kcal} / \mathrm{mol})\end{array}$} & \multirow[b]{2}{*}{$\begin{array}{c}\text { GSH } \\
\text { conjugates }\end{array}$} \\
\hline & $\begin{array}{c}\text { Total } \\
\text { Electronic } \\
\text { Energy } \\
\text { (Hartree) }\end{array}$ & $\begin{array}{c}\text { Thermal } \\
\text { correction for } \\
\text { enthapy } \\
\text { (Hartree) }\end{array}$ & $\begin{array}{c}\text { Total } \\
\text { Electronic } \\
\text { Energy } \\
\text { (Hartree) }\end{array}$ & $\begin{array}{c}\text { Thermal } \\
\text { correction for } \\
\text { enthapy } \\
\text { (Hartree) }\end{array}$ & & \\
\hline 13 & -361.277266 & 0.121145 & -361.903342 & 0.136537 & 0 & +++ \\
\hline 14 & -400.463037 & 0.152563 & -401.08656 & 0.167913 & -1.58 & ++ \\
\hline 15 & -591.916539 & 0.230258 & -592.545679 & 0.245752 & 1.86 & +++ \\
\hline 16 & -432.519252 & 0.127883 & -433.148442 & 0.143163 & 2.02 & + \\
\hline 17 & -377.306676 & 0.108746 & -377.936514 & 0.124319 & 2.25 & BLD \\
\hline 18 & -393.297014 & 0.095507 & -393.941107 & 0.111979 & 10.63 & BLD \\
\hline 19 & -432.485852 & 0.126911 & -433.120248 & 0.143152 & 4.69 & BLD \\
\hline 20 & -643.285317 & 0.265027 & -643.927166 & 0.265027 & 9.37 & BLD \\
\hline
\end{tabular}

${ }^{\mathrm{a}} \triangle \mathrm{BDEs}$ of first hydrogen atom abstraction refers to the difference between the lowest BDE of any N-H or $\mathrm{O}-\mathrm{H}$ of a fragment vs. the $\mathrm{O}-\mathrm{H}$ bond of 4-hydroxyaniline which has a lower BDE than $\mathrm{N}-\mathrm{H}$ bond of $\mathrm{N}$ methylbenzene-1,4-diamine. 
Table S6. Total electronic energies, thermal corrections, and calculated $\triangle B D E s$ for the parent and radical of entries 21 to 35

\begin{tabular}{|c|c|c|c|c|c|c|}
\hline \multirow[b]{2}{*}{ Entry } & \multicolumn{2}{|c|}{ Radical } & \multicolumn{2}{|c|}{ Parent } & \multirow[b]{2}{*}{$\begin{array}{c}\Delta \mathrm{BDE}^{\mathrm{a}} \\
(\mathrm{kcal} / \mathrm{mol})\end{array}$} & \multirow[b]{2}{*}{$\begin{array}{c}\text { GSH } \\
\text { conjugates }\end{array}$} \\
\hline & $\begin{array}{c}\text { Total } \\
\text { Electronic } \\
\text { Energy } \\
\text { (Hartree) }\end{array}$ & $\begin{array}{l}\text { Thermal } \\
\text { correction for } \\
\text { enthapy } \\
\text { (Hartree) }\end{array}$ & $\begin{array}{c}\text { Total } \\
\text { Electronic } \\
\text { Energy } \\
\text { (Hartree) }\end{array}$ & $\begin{array}{c}\text { Thermal } \\
\text { correction for } \\
\text { enthapy } \\
\text { (Hartree) }\end{array}$ & & \\
\hline 21 & -572.045123 & 0.243227 & -572.696039 & 0.259119 & 0 & ++ \\
\hline 22 & -588.074101 & 0.231078 & -588.729468 & 0.24694 & 2.81 & + \\
\hline 23 & -588.077143 & 0.230905 & -588.729598 & 0.247014 & 0.83 & + \\
\hline 24 & -604.097364 & 0.218884 & -604.760611 & 0.234514 & 7.9 & BLD \\
\hline 25 & -604.10986 & 0.218813 & -604.762847 & 0.234649 & 1.33 & + \\
\hline 26 & -604.105981 & 0.218652 & -604.759355 & 0.234377 & 1.65 & ++ \\
\hline 27 & -604.073337 & 0.218054 & -604.731179 & 0.234398 & 4.06 & BLD \\
\hline 28 & -671.118883 & 0.235539 & -671.772432 & 0.251429 & 1.65 & ++ \\
\hline 29 & -1031.101164 & 0.234106 & -1031.756465 & 0.250111 & 2.68 & ++ \\
\hline 30 & -3143.947252 & 0.233717 & -3144.603535 & 0.249715 & 3.3 & +++ \\
\hline 31 & -908.501316 & 0.25245 & -909.154032 & 0.268309 & 1.15 & ++ \\
\hline 32 & -908.495664 & 0.251884 & -909.154254 & 0.26797 & 4.69 & +++ \\
\hline 33 & -611.247247 & 0.274166 & -611.89724 & 0.290061 & -0.58 & +++ \\
\hline 34 & -611.241149 & 0.273621 & -611.895214 & 0.289581 & 1.93 & + \\
\hline 35 & -627.280178 & 0.262581 & -627.932619 & 0.27828 & 1.08 & + \\
\hline
\end{tabular}

${ }^{\mathrm{a}} \triangle \mathrm{BDEs}$ of first hydrogen atom abstraction refers to the difference between the lowest BDE of any N-H of a morpholine fragment vs. N-H bond of the unsubstituted 1,4-morpholine aminobenzene. 
For LUMO and HOMO calculations, the structures were first optimized using Gaussian, ${ }^{5}$ with either unrestricted (for radicals) or restricted (for quinoid intermediates) HF/6-311+G** in water solvent (PCM). ${ }^{6}$ A single point energy calculation was performed at the end using unrestricted (for radicals) or restricted (for quinoid intermediates) MP2/6-311+G** with PCM water solvent model for the HF optimized conformations.

The following legend applies to Figures S1-S3:

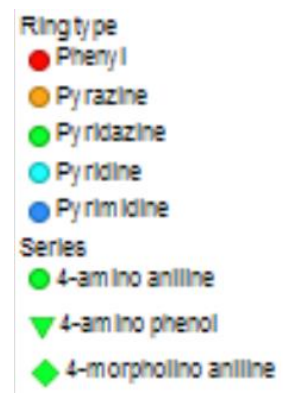

Figure S1. Plot of the LUMO energy for quinoid intermediates versus GSH rank

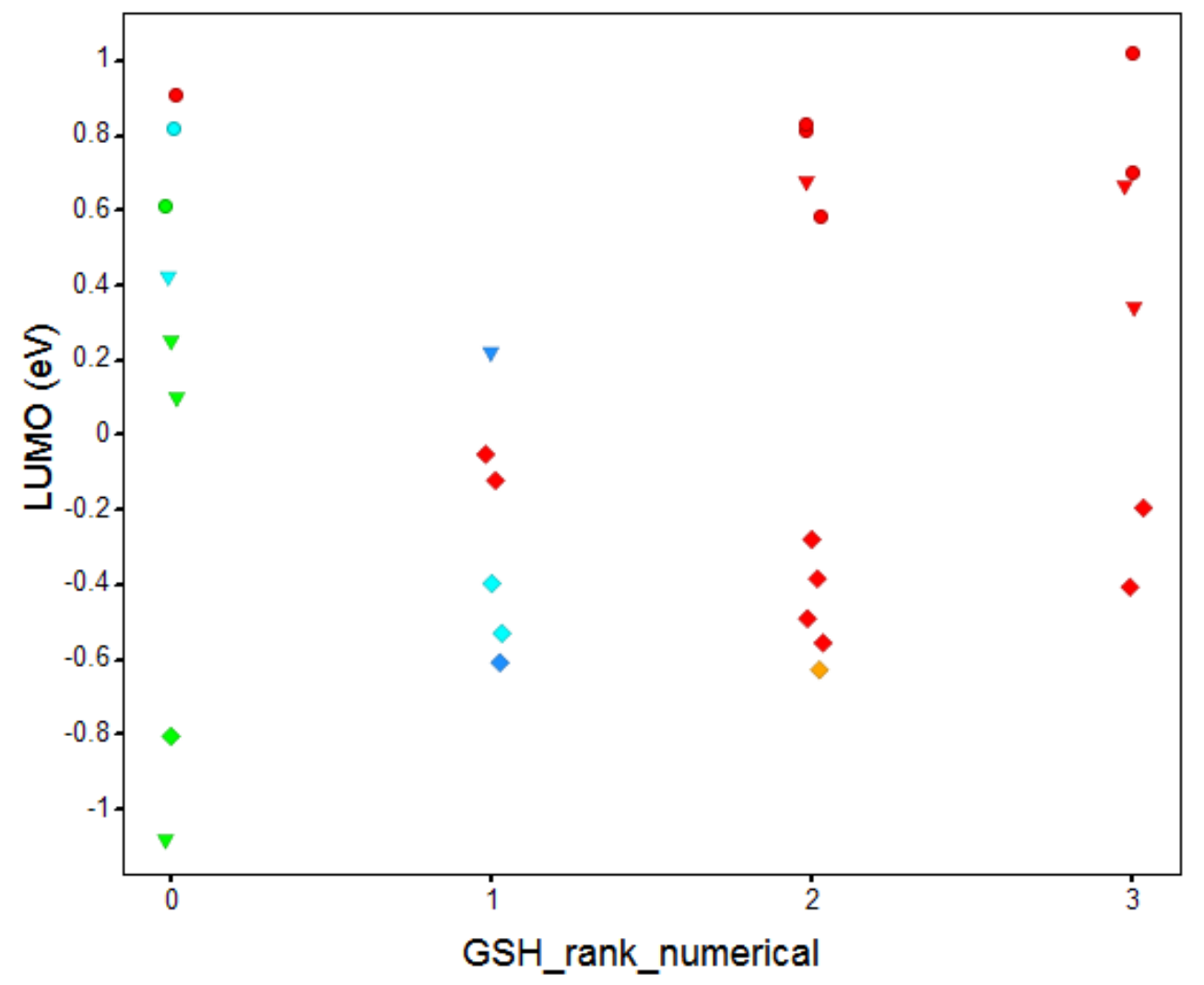

GSH_rank_numerical of $0,1,2$, and 3 correspond to BLD,,+++ , and +++ , respectively. BLD", below limit of detection; "+" represents a range from $10^{3}-10^{5}$; "++": $10^{5}-10^{6}$; “+++": $>10^{6}$ cps. For assumed structures of quinoid intermediates, see below. 
Figure S2. Plot of the HOMO energy for quinoid intermediates versus GSH rank

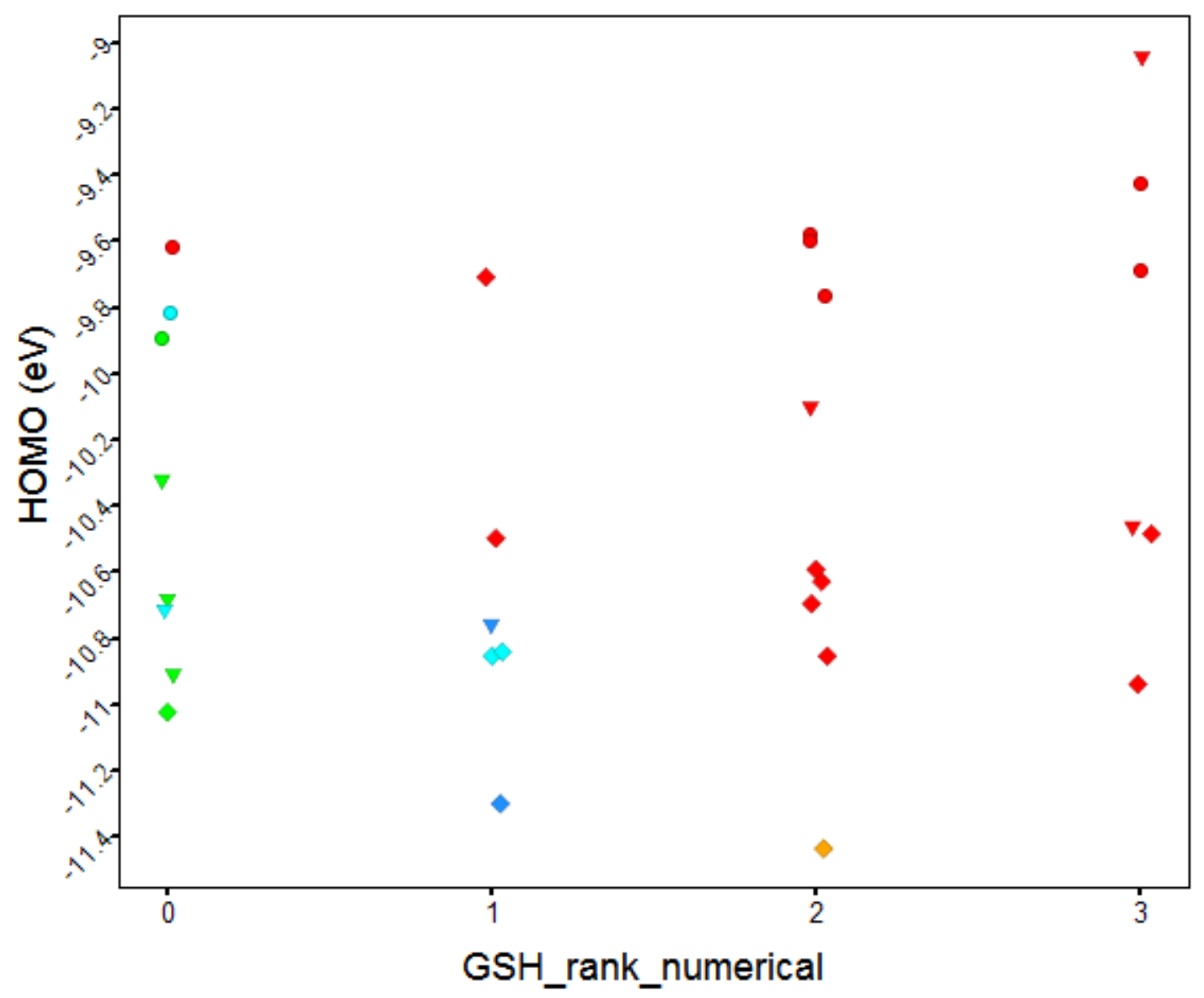

GSH_rank_numerical of $0,1,2$, and 3 correspond to BLD,,+++ , and +++ , respectively. BLD", below limit of detection; "+" represents a range from $10^{3}-10^{5}$; “++": $10^{5}-10^{6}$; “+++": $>10^{6}$ cps. For assumed structures of quinoid intermediates, see below. 
Figure S3. Plot of the HOMO-LUMO gap energy for quinoid intermediates versus GSH rank

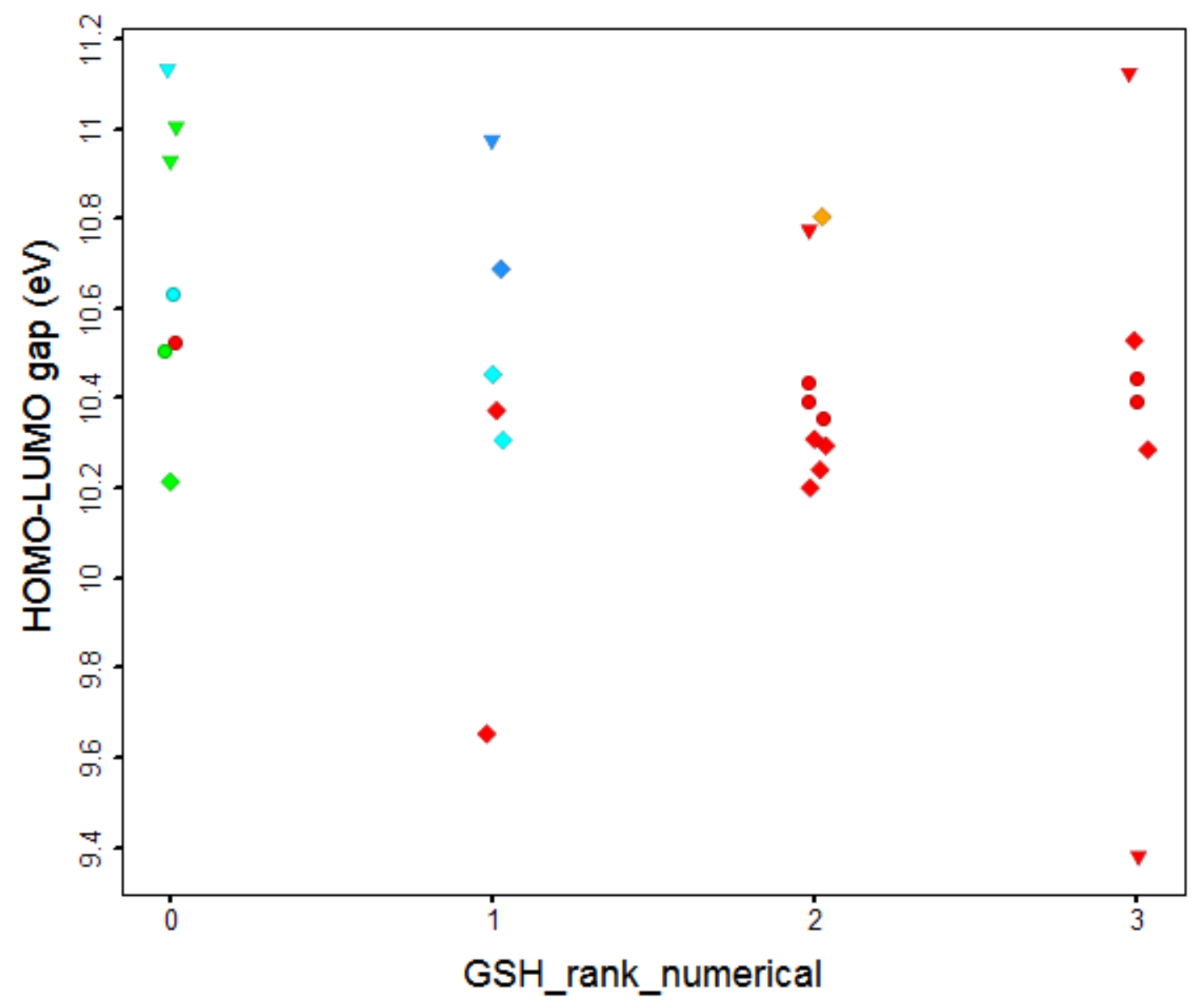

GSH_rank_numerical of $0,1,2$, and 3 correspond to BLD,,+++ , and +++ , respectively. BLD”, below limit of detection; "+" represents a range from $10^{3}-10^{5}$; “++": $10^{5}-10^{6}$; “+++": $>10^{6}$ cps. For assumed structures of quinoid intermediates, see below. 
Quinoid structures used for Figures S1-S3:

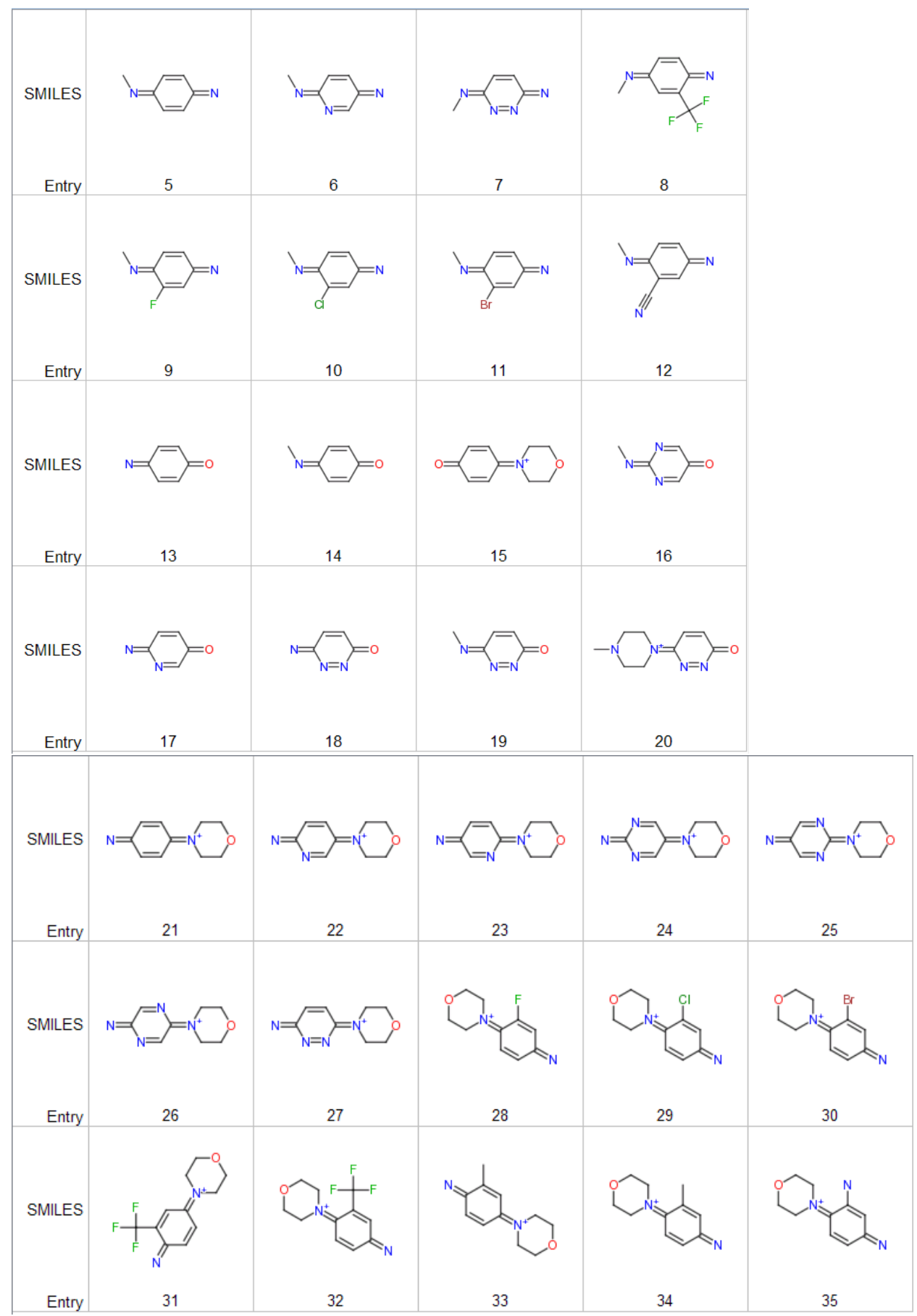




\section{Supporting Information References}

1) Zhang, C.; Kenny, J. R.; Le, H.; Deese, A.; Ford, K. A.; Lightning, L. K.; Fan, P. W.; Driscoll, J. P.; Halladay, J. S.; Hop, C. E.; Khojasteh, S. C. Novel mechanism for dehalogenation and glutathione conjugation of dihalogenated anilines in human liver microsomes: evidence for ipso glutathione addition. Chem. Res. Toxicol. 2011, 24, 1668-1677.

2) Zhang, C.; S., M.; Delarosa, E. M.; Tay, S.; Sodhi, J.; Musinipally, V.; Chang, P.; Pai, R.; Halladay, J. S.; Misner, M.; Kenny, J. R.; Hop, C. E.; Khojasteh, C. For a series of methylindole analogs, reactive metabolite formation is a poor predictor of intrinsic cytotoxicity in human hepatocytes. Toxicol. Res. 2014, 3, 184-190.

3) Maestro; Schrödinger, Inc.: New York, 2017.

4) Sellers, B. D.; James, N. C.; Gobbi, A. A Comparison of Quantum and Molecular Mechanical Methods to Estimate Strain Energy in Druglike Fragments. J. Chem. Inf. Model. 2017, 57, 12651275.

5) Gaussian 09, Revision E.01, M. J. Frisch, G. W. Trucks, H. B. Schlegel, G. E. Scuseria, M. A. Robb, J. R. Cheeseman, G. Scalmani, V. Barone, B. Mennucci, G. A. Petersson, H. Nakatsuji, M. Caricato, X. Li, H. P. Hratchian, A. F. Izmaylov, J. Bloino, G. Zheng, J. L. Sonnenberg, M. Hada, M. Ehara, K. Toyota, R. Fukuda, J. Hasegawa, M. Ishida, T. Nakajima, Y. Honda, O. Kitao, H. Nakai, T. Vreven, J. A. Montgomery, Jr., J. E. Peralta, F. Ogliaro, M. Bearpark, J. J. Heyd, E. Brothers, K. N. Kudin, V. N. Staroverov, T. Keith, R. Kobayashi, J. Normand, K. Raghavachari, A. Rendell, J. C. Burant, S. S. Iyengar, J. Tomasi, M. Cossi, N. Rega, J. M. Millam, M. Klene, J. E. Knox, J. B. Cross, V. Bakken, C. Adamo, J. Jaramillo, R. Gomperts, R. E. Stratmann, O. Yazyev, A. J. Austin, R. Cammi, C. Pomelli, J. W. Ochterski, R. L. Martin, K. Morokuma, V. G. Zakrzewski, G. A. Voth, P. Salvador, J. J. Dannenberg, S. Dapprich, A. D. Daniels, O. Farkas, J. B. Foresman, J. V. Ortiz, J. Cioslowski, and D. J. Fox, Gaussian, Inc., Wallingford CT, 2013.

6) Frisch, M. J.; Pople, J. A.; Binkley, J. S. Self-Consistent Molecular-Orbital Methods .25. Supplementary Functions for Gaussian-Basis Sets. J. Chem. Phys. 1984, 80, 3265-3269. 
7) Tomasi, J.; Mennucci, B.; Cammi, R. Quantum mechanical continuum solvation models. Chem. Rev. 2005, 105, 2999-3093.

8) Lee, C.; Yang, W.; Parr, R. G. Development of the Colle-Salvetti correlation-energy formula into a functional of the electron density. Phys. Rev. B 1988, 37, 785-789.

9) Becke, A. D. Density-functional thermochemistry. III. The role of exact exchange. J. Chem. Phys. 1993, $98,5648-5652$. 